\title{
Brown and beige adipose tissue regulate systemic metabolism to resist diet-induced obesity through metabolite signals in an inter-organ signaling axis
}

Anna Whitehead

University of Leeds

Fynn Krause

University of Cambridge https://orcid.org/0000-0001-6664-2637

Amy Moran

University of Leeds

Jason Scragg

University of Leeds

Ben McNally

University of Cambridge

Edward Boateng

University of Leeds

Steven Murfitt

University of Cambridge

Sam Virtue

University of Cambridge

John Wright

University of Leeds

Amanda MacCannell

University of Leeds

Jack Garnham

University of Leeds

Graeme Davies

Medlmmune

James Dodgson

Medlmmune

Jurgen Schneider

University of Leeds

Andrew Murray

University of Cambridge

Christopher Church 
Medlmmune

\section{Antonio Vidal-Puig}

University of Cambridge https://orcid.org/0000-0003-4220-9577

\section{Klaus Witte}

University of Leeds https://orcid.org/0000-0002-7146-7105

Julian Griffin

Medical Research Council

\section{Lee Roberts ( $\sim$ L.D.Roberts@leeds.ac.uk )}

University of Leeds https://orcid.org/0000-0002-1455-5248

\section{Article}

Keywords: Brown and beige adipose tissue, Metabolism, 3-methyl-2-oxovaleric acid (MOVA), 5-oxoproline (50P), $\beta$-hydroxyisobutyric acid (BHIBA)

Posted Date: July 7th, 2020

DOl: https://doi.org/10.21203/rs.3.rs-38426/v1

License: (c) (7) This work is licensed under a Creative Commons Attribution 4.0 International License. Read Full License

Version of Record: A version of this preprint was published at Nature Communications on March 26th, 2021. See the published version at https://doi.org/10.1038/s41467-021-22272-3. 


\section{Abstract}

Brown and beige adipose tissue are emerging as distinct endocrine organs. These tissues are functionally associated with skeletal muscle, adipose tissue metabolism and systemic energy expenditure, suggesting an inter-organ signaling network. Using a metabolomic approach we identified 3-methyl-2-oxovaleric acid (MOVA), 5-oxoproline (5OP), and $\beta$-hydroxyisobutyric acid (BHIBA) as small molecule metabokines synthesized in browning adipocytes and secreted via monocarboxylate transporters. MOVA, 5OP and BHIBA induce a brown adipocyte-specific phenotype in white adipocytes and mitochondrial oxidative energy metabolism in skeletal myocytes both in vitro and in vivo. MOVA and 5OP signal through cAMPPKA-p38 MAPK and BHIBA via mTOR. These metabolites reduce adiposity, increase energy expenditure and improve glucose and insulin homeostasis in mouse models of obesity and diabetes. In humans, plasma and adipose tissue MOVA, 5OP and BHIBA concentrations are correlated with markers of adipose browning and inversely associated with BMI. Our findings identify beige adipose-brown adipose-muscle physiological metabokine crosstalk.

\section{Introduction}

Brown adipose tissue (BAT) functions to regulate body temperature through non-shivering thermogenesis; the dissipation of chemical energy to produce heat ${ }^{1,2}$. Beige adipocytes are interspersed within the white adipose tissue (WAT) of rodents and humans, and can be induced to switch from a white adipocyte-like phenotype to a brown adipocyte-like phenotype; a process known as "browning" ${ }^{3}$. Brown and activated beige cells are characterized by high levels of fatty acid $\beta$-oxidation, mitochondrial content and thermogenesis ${ }^{4}$. Thermogenesis occurs through the activity and increased expression of several specific gene products, including uncoupling protein 1 (UCP1), an inner mitochondrial membrane protein that uncouples substrate oxidation from ATP synthesis to generate heat ${ }^{5}$. Activated brown and beige adipocytes alter systemic energy metabolism, increasing substrate oxidation and energy expenditure, with potential for therapeutic exploitation against metabolic diseases including Type 2 Diabetes Mellitus (T2DM) and obesity ${ }^{6,7}$.

The effects of BAT and beige adipose tissue on energy balance may not solely depend on the action of UCP1. Activation of thermogenesis in brown and beige adipose tissues may lead to propagation of thermogenesis in surrounding adipocytes and distal adipose depots. Transplantation studies of both beige and brown fat in mice suggest that these tissues can signal to activate endogenous beige and brown fat and improve glucose homeostasis in skeletal muscle ${ }^{8,9}$. In murine models of both adipose tissue browning and increased BAT thermogenesis, fatty acid oxidation in skeletal muscle is increased ${ }^{8-}$ 10. The anti-obesity and anti-diabetic effects of brown and beige adipose tissues are also not solely reliant on the thermogenic process. Mice lacking UCP1 are resistant to diet-induced obesity at room temperature, yet mice lacking brown/beige fat are highly susceptible to an obese phenotype ${ }^{11-15}$. Therefore, beige and brown fat may influence systemic metabolism through non-UCP1 thermogenic mechanisms, potentially mediated through the release of endocrine signals in the adipocyte "secretome". 
In this study a discrete panel of small molecule metabolite paracrine and endocrine signals, secreted from both beige and brown adipocytes are identified. These metabokines increase adipose tissue, skeletal muscle and systemic energy metabolism. We propose these brown and beige adipokine-like small molecules function in an adipose-adipose and adipose-skeletal muscle inter-organ signaling axis.

\section{Results}

\section{Metabolite signals from browning adipocytes increase expression of brown adipocyte-associated genes in primary adipocytes}

Adipocyte browning was induced in primary adipocytes differentiated from the stromal vascular fraction of subcutaneous (inguinal) WAT of mice using two distinct canonical signaling mechanisms, an adenylate cyclase activator (forskolin), and Peroxisome Proliferator-Activated Receptor $\delta$ (PPAR $\delta$ ) agonist $(G W 0742)^{16}$. Cells were washed and fresh serum-free media was conditioned on the cells for $24 \mathrm{hr}$. Conditioned media was transferred to naïve primary adipocytes (Fig. 1a) and induced expression of brown adipocyte-associated genes in naive primary adipocytes (Fig. 1b \& 1c). Expression of brownadipocyte associated genes, including Ucp1, peroxisome proliferator-activated receptor $y$ co-activator1a (Ppargc1a; Pgc1a), cell death-inducing DFFA-like effector a (Cidea), carnitine palmitoyltransferase 1b (Cpt1b), acyl-CoA dehydrogenase very long chain (Acadvl) and cytochrome $C$ (Cycs) was further enhanced following media protein denaturation by boiling, implicating a non-protein small molecule mediator(s) (Fig. 1b \& c). These data may also indicate the presence of a secreted protein inhibitor of browning. To define the physicochemical nature of the small molecule mediators, aqueous-soluble metabolites were extracted from media conditioned on activated beige adipocytes using solvent partition. The aqueous soluble metabolites were reconstituted in fresh media and transferred to naïve primary adipocytes (Fig 1d). Expression of brown-adipocyte associated genes was induced by aqueous-soluble metabolites released from browning adipocytes.

To identify candidate metabolites that may induce browning, we applied both Gas Chromatography Mass Spectrometry (GC-MS) and Liquid Chromatography-Mass Spectrometry (LC-MS) metabolic profiling to media conditioned on browning adipocytes. GW0742 and forskolin were not detected in conditioned media. We focused on identifying metabolites commonly enriched in both PPAR $\delta$ agonism and forskolintreated adipocyte media to determine common species. Multivariate statistical models of the metabolic profiling data identified common metabolite species enriched in the media by both cAMP and PPAR $\delta$ induced browning (Fig. 1e). The concentration of sugar species and the branched-chain amino acids (BCAAs) valine and isoleucine was decreased in the media of browning adipocytes (Fig 1f). Concomitantly the concentration of 5-oxoproline (5OP) and the BCAA catabolites a-hydroxyisocaproic acid (HIC), a-ketoisovaleric acid (AKV), a-hydroxyisovaleric acid (AHI), 3-methyl-2-oxovaleric acid (MOVA), $\beta$-hydroxyisobutyric acid (BHIBA) and $\beta$-hydroxyisovaleric acid (BHIVA) was increased in the media. Glycerol, a marker of lipolysis, was also increased. 
Next, we examined whether physiological plasma concentrations of the BCAA metabolites and 50P

increased the expression of brown adipocyte-associated genes in primary adipocytes (Fig. 1g) ${ }^{17-20}$. Physiological plasma concentrations of the metabolites are given in supplementary table 1. MOVA, 5OP, BHIBA and BHIVA significantly and robustly induced expression of brown adipocyte-associated genes including Ucp 1, Cidea and Cpt1b. The media of primary mouse canonical brown adipocytes treated with either forskolin $(1 \mu \mathrm{M})$ or the PPARठ agonist $(100 \mathrm{nM})$ was also analyzed using our metabolomics approach (Fig. 1h). MOVA, 5OP, BHIBA and BHIVA were also enriched in the media of primary brown adipocytes following cAMP or PPARס-mediated induction of brown adipocyte-associated gene expression (Fig. 1h \& i).

Therefore, the metabolites MOVA, 5OP, BHIBA and BHIVA are released from primary white and brown adipocytes in response to thermogenic stimuli and stimulate the expression of brown-adipocyte associated genes in naïve adipocytes.

\section{Metabolite signals are secreted from browning human adipocytes and induce a brown adipocyte-like functional phenotype}

We determined whether the secretion of the candidate metabokines from browning adipocytes was conserved in human cells. Human primary adipocytes were treated with either forskolin or a PPARס agonist to induce a brown-adipocyte like phenotype (Supplementary Fig. 1a - h). MOVA, 5OP, BHIBA and BHIVA were enriched in the media of forskolin $(1 \mu \mathrm{M})$ and PPAR $\delta$ agonist (100 nM GW0742) treated human adipocytes (Fig. 2a). Treatment of primary human adipocytes with physiological plasma concentrations (Supplementary Table 1) of MOVA $(20 \mu \mathrm{M}), 50 \mathrm{P}(20 \mu \mathrm{M})$, BHIBA $(20 \mu \mathrm{M})$ and BHIVA (10 $\mu \mathrm{M})$ induced expression of a panel of brown adipocyte-associated genes including UCP1, CIDEA and $P G C 1 a$ (Fig. 2b). Induction of UCP1 expression in primary human adipocytes treated with metabolites in the physiological micromolar range occurred in a dose-dependent manner (Supplementary Fig $2 \mathrm{a}-\mathrm{d}$ ). To determine whether changes in the expression of brown adipocyte-associated genes were translated to the level of protein, the concentrations of UCP1 protein in metabokine-treated human primary adipocytes were determined by ELISA (Fig 2c). We further investigated whether the metabolites induced functional effects consistent with browning on energy expenditure in human primary adipocytes. Both the basal and succinate-stimulated (complex II) oxygen consumption rates of adipocytes treated with MOVA $(20 \mu \mathrm{M})$, $50 \mathrm{P}(20 \mu \mathrm{M}), \mathrm{BHIBA}(20 \mu \mathrm{M})$ and BHIVA $(10 \mu \mathrm{M})$ were increased (Fig. 2d). Primary human adipocytes were treated with MOVA $(20 \mu \mathrm{M}), 50 \mathrm{P}(20 \mu \mathrm{M})$, BHIBA $(20 \mu \mathrm{M})$ and BHIVA $(10 \mu \mathrm{M})$ and incubated in serum-free media containing $\mathrm{U}^{13} \mathrm{C}$-palmitate to monitor adipocyte fatty acid $\beta$-oxidation. The labeled palmitate is catabolized via $\beta$-oxidation, releasing labeled acetyl-CoA, which enters the TCA cycle (Supplementary Fig 2e). GC-MS analysis identified increased relative enrichment of downstream TCA cycle metabolites citrate, fumarate, and malate in MOVA, 5OP and BHIBA-treated adipocytes (Fig. $2 \mathrm{e}-\mathrm{g}$ ), confirming fatty acid $\beta$-oxidation is increased in these cells. Browning is accompanied by an increase in glucose and fatty acid uptake into adipocytes. The uptake of glucose and fatty acid into human primary adipocytes treated with the metabolites was measured with the fluorescent glucose analog 6-NDBG or the fluorescent fatty acid analog BODIPY-FA (Fig. 2h \& i) (Supplementary Fig $2 f-$ m). The metabolites 
increased adipocyte glucose and fatty acid uptake. We sought to further characterize the transcriptional programme induced in adipocytes by the candidate metabolite signals, and to confirm that the effects on brown adipocyte-associated gene expression are conserved in an independent in vitro model of human adipose tissue. A gene expression array of key adipocyte and brown adipocyte-associated genes was used to probe immortalized human white preadipocytes isolated from neck fat and differentiated to mature adipocytes in the presence of MOVA $(20 \mu \mathrm{M})$ (Supplementary Table 2), 5OP $(20 \mu \mathrm{M})$ (Supplementary Table 3), BHIBA ( $20 \mu \mathrm{M})$ (Supplementary Table 4) or BHIVA (10 $\mu \mathrm{M})$ (Supplementary Table 5). Confocal imaging of immortalized human adipocytes treated with the candidate metabokines identified MOVA, 5OP and BHIBA significantly increased cellular UCP1 protein content (Fig. 2j \& k). Functionally, leak respiration and electron transport chain uncoupling were increased in immortalized human adipocytes treated with the metabolites (Fig 2l-o).

These data indicate that MOVA, 5OP, BHIBA and, to a lesser extent, BHIVA induce gene and protein expression and a functional phenotype consistent with browning in two human adipocyte models.

\section{Transcriptional analysis and ${ }^{13} \mathrm{C}$-isotope substrate tracing reveal mechanisms of metabokine biosynthesis and secretion by browning adipocytes}

BCAAs were depleted in the media of browning adipocytes (Fig 1f). MOVA, BHIBA and BHIVA are generated through the degradation of the BCAAs isoleucine, valine and leucine, respectively. These pathways share multiple enzymes. 5OP is synthesized from glutamate. We examined mechanisms by which adipocyte browning may increase the concentrations of MOVA, BHIBA, BHIVA and $50 \mathrm{P} . \mathrm{U}-{ }^{13} \mathrm{C}-$ labeled isoleucine, valine, leucine, and glutamate were used to monitor stable isotope enrichment through the biosynthetic pathways and into extracellular accumulation of the candidate metabokines produced by human primary adipocytes treated with forskolin. Concomitantly, we performed RNA-Seq on human primary adipocytes treated with forskolin. Induction of the browning response increased both the intracellular and extracellular (culture media) ${ }^{13} \mathrm{C}$-enrichment of MOVA (Supplementary Fig. 3a - d), BHIBA (Supplementary Fig. 3e - j), BHIVA (Supplementary Fig. 3k - n) and 5OP (Supplementary Fig. 3o u). The expression of the BCAA catabolic enzymes, branched chain amino acid transaminase 2 (BCAT2), branched chain keto acid dehydrogenase $\mathrm{E} 1$ subunit beta $(B C K D H B)$, acyl-CoA dehydrogenase short chain (ACADS), acyl-CoA dehydrogenase medium chain (ACADM), Enoyl-Coenzyme A, Hydratase/3Hydroxyacyl Coenzyme A Dehydrogenase (EHHADH), hydroxyacyl-CoA dehydrogenase (HADHA) and Enoyl-CoA Hydratase, Short Chain 1 (ECHS1) was increased in forskolin-treated adipocytes (Supplementary Fig. $3 a-n$ ). The expression of the 50P biosynthetic enzymes glutathione synthetase (GSS), $\gamma$-glutamyltransferase 7 (GGT7) and $\gamma$-glutamylcyclotransferase (GGCT) were also increased in browning adipocytes (Supplementary Fig. $30-u$ ).

These data identify that browning induces a transcriptional program upregulating expression of the metabokine biosynthetic enzymes and driving adipocyte synthesis and release of MOVA, 5OP, BHIBA and BHIVA. 


\section{Metabokine signals are exported from browning adipocytes via monocarboxylate transporters}

Next, we investigated the mechanisms through which the browning adipocytes export the metabolite signals. MOVA, 5OP, BHIBA and BHIVA, structurally, share a common single carboxyl group. Our RNA-seq analysis identified that monocarboxylate transporter1 (MCT1) expression was increased in human primary adipocytes treated with forskolin (SLC16A1, Log Fold-change $=0.37, P<0.05, n=3$ ). MCT1 functions to both export and import monocarboxylates through the plasma membrane. We used a pharmacological MCT inhibitor (a-cyano-4-hydroxycinnamate) to determine the involvement of MCTs in browning-mediated secretion of the metabokines. Inhibition of MCTs abrogated forskolin-induced secretion of the metabokine signals, decreasing MOVA, 5OP, BHIBA and BHIVA extracellular concentration whilst increasing their intracellular concentration (Figure $3 a-d)$ ). Therefore MCTs are required for metabokine export from browning adipocytes.

\section{MOVA, 50P and BHIBA regulate metabolism in skeletal myocytes}

In murine models of both adipose tissue browning and BAT activity, fatty acid oxidation in skeletal muscle is increased ${ }^{8-10}$, representing an adipose-muscle metabolic signaling axis. We hypothesized that the metabolites secreted from browning adipocytes may contribute to the functional link between browning adipose tissue and muscle. First, we reconstituted this adipose tissue-muscle functional relationship in vitro. As previously described, conditioned serum-free media was collected from primary mouse adipocytes treated with forskolin $(1 \mu \mathrm{M})$. Conditioned media was transferred to mouse $\mathrm{C} 2 \mathrm{C} 12$ myotubes and induced expression of transcriptional regulators of metabolism (Ppara, Pgc1a), fatty acid $\beta$-oxidation genes including $C p t 1 b$, and $A c a d v l$, and mitochondrial genes $C y c s$ and respiratory chain complex 1 component NADH:Ubiquinone Oxidoreductase Core Subunit S1 (Ndufs1) (Fig. 4a). The metabolites MOVA and 5OP robustly induced expression of the metabolic gene panel in mouse myotubes (Fig. 4b).

The adipose tissue-muscle in vitro signaling model was translated to human primary cells. Conditioned media from browning human adipocytes induced expression of key fatty acid metabolism genes in human myocytes (Fig. 4c). The effect of MOVA and 50P on metabolic, mitochondrial, and fatty acid oxidation gene expression was conserved in human primary skeletal myocytes and was dose responsive in the physiological low micromolar range (Fig. 4d) (Supplementary Fig 4a-d). BHIBA was also observed to increase expression of PPARa and $C P T 1 b$ in human primary skeletal myocytes. To confirm that transcriptional changes in human myocytes are accompanied by a dose-dependent change in functional phenotype, the oxygen consumption rates of primary myocytes treated with MOVA ( $5 \mu \mathrm{M}$ and $20 \mu \mathrm{M})$, 5OP $(5 \mu \mathrm{M}$ and $20 \mu \mathrm{M}), \mathrm{BHIBA}(5 \mu \mathrm{M}$ and $20 \mu \mathrm{M})$ and BHIVA $(2.5 \mu \mathrm{M}$ and $10 \mu \mathrm{M})$ were measured. Basal respiration rates of the myocytes were increased by MOVA (Supplementary Fig. 4e), 5OP (Supplementary Fig. 4f) and BHIBA (Supplementary Fig. 4g), but not BHIVA (Supplementary Fig. 4h). MOVA and 5OP induced the greatest increase in oxidative gene expression in both mouse and human primary myocytes. These metabolites were selected for characterization in primary myocytes using a substrate-inhibitor high-resolution respirometry protocol. MOVA and 50P increased respiratory capacity in permeabilized 
human myocytes supported by substrates for mitochondrial complex I (glutamate and malate), fatty acid $\beta$-oxidation (octanoylcarnitine) and mitochondrial complex II (succinate) respiration (Fig $4 \mathrm{e} \& \mathrm{f}$ ). $50 \mathrm{P}$ also increased maximal electron transport chain capacity (FCCP-uncoupled) in myocytes (Fig 4f). Murine models of adipose browning and thermogenesis activate fatty acid and glucose catabolism in skeletal muscle ${ }^{8,9,11}$. Therefore we investigated the effect of the metabokines on uptake of both glucose and fatty acid into human primary myocytes using the fluorescent glucose analog 6-NDBG and the fluorescent fatty acid analog BODIPY-FA, respectively (Fig. 4h \& i) (Supplementary Fig. $4 i-n$ ).

These analyses identify that MOVA, 5OP, and to a lesser extent BHIBA, regulate both murine and human skeletal myocyte metabolism consistent with an adipose-muscle metabolic signaling axis.

\section{Metabolite signals are enriched by cold conditioning and depleted by obesity in vivo}

To determine if MOVA, 5OP, BHIBA and BHIVA function as brown and beige adipocyte metabokines in vivo we examined their concentrations in the BAT, subcutaneous inguinal WAT and blood plasma of physiological models of increased and decreased adipose thermogenic function. We examined mice housed at thermoneutrality, room temperature and under thermogenic conditions with cold exposure at $8^{\circ} \mathrm{C}$ for a period of one week and one month. As expected, cold exposure robustly induced a thermogenic phenotype in mouse BAT and subcutaneous WAT gene expression, UCP1 protein expression and adipocyte morphology (Supplementary Fig. 5a - f). The concentrations of MOVA, 5OP, BHIBA and BHIVA were increased in the BAT and subcutaneous WAT of cold challenged mice (Fig. 5a \& b). Consistent with the increase in the metabolite concentrations in the tissues, the expression of the MOVA, BHIBA and BHIVA biosynthetic genes (Bcat2, Bckdhb, Acads, Acadm, Ehhadh, Hadha and Echs1) and the 5OP biosynthetic genes (Gss, Ggct) were increased in the BAT (Fig. 5c) and subcutaneous WAT (Fig. 5d) of cold challenged mice. The expression of the monocarboxylate transporter, Mct1, was also induced by cold challenge in the BAT (Fig. 5c) and subcutaneous WAT (Fig. 5d) of mice.

In line with their potential as secreted brown and beige adipocyte paracrine and endocrine metabokines, plasma concentrations of the metabolites were also increased in mice housed in a cold environment (Fig. $5 e)$.

Brown and beige adipose tissue are lost during the so called "whitening" effect associated with obesity, leading BAT to morphologically and metabolically resemble WAT (22). We determined if the metabolites MOVA, 5OP, BHIBA and BHIVA are decreased by diet-induced obesity. Diet-induced obese mice, fed a $60 \%$ fat diet for 17 weeks, had greater body weight and impaired glucose tolerance compared to matched chow-fed controls (Supplementary Fig. $5 \mathrm{~g} \& 5 \mathrm{~h}$ ). Obese mice exhibited markers of whitening within their intrascapular BAT, with decreased expression of thermogenic genes (Supplementary Fig. 5i) and a whiteadipocyte like morphology (Supplementary Fig. 5j). In agreement with these observations, the BAT concentrations of MOVA, 5OP, BHIBA and BHIVA were decreased by diet-induced obesity (Supplementary Fig. 5k). 
Therefore the metabokine signals are modulated in adipose depots and systemically in in vivo physiological models of altered thermogenic function.

\section{The metabokines MOVA, 5OP and BHIBA increase systemic energy expenditure and regulate the adipose tissue and skeletal muscle metabolic phenotype in vivo}

Browning of WAT and activation of BAT thermogenesis may regulate metabolism in WAT and skeletal muscle through secreted small molecule signals which alter systemic energy balance. MOVA, 50P, BHIBA and BHIVA are secreted from browning adipocytes, increase in adipose tissue and plasma in vivo in response to canonical thermogenesis activated by cold, decrease in response to thermoneutrality and BAT "whitening", and regulate browning and fatty acid metabolism in adipocytes and myocytes respectively. We investigated the effect of MOVA, 5OP, BHIBA and BHIVA on the in vivo metabolic phenotype of mice. Six-week-old mice fed standard chow were either treated with MOVA (100 mg/kg/day), 5OP (100 mg/kg/day), BHIBA (150 mg/kg/day) or BHIVA ( $125 \mathrm{mg} / \mathrm{kg} /$ day) in drinking water for 17 weeks (based on preliminary dose escalation studies) or remained untreated (control mice). Treatment increased the plasma concentrations of the metabolites in the mice within the low micromolar physiological range (Supplementary Fig $6 a-d$ ). Water intake was not different between groups (Supplementary Fig 6e). Weight gain of 5OP and MOVA treated mice was decreased compared with controls (Supplementary Fig 6f). Analysis with metabolic cages indicated BHIBA, MOVA and 5OP increased energy expenditure (Supplementary Fig $6 \mathrm{~g}-\mathrm{j}$ ) and oxygen consumption (Supplementary Fig 6 $k-n$ ) independent of body mass. Metabolite treatment did not affect the activity of the mice (Supplementary Fig 60). Food intake was increased in the 50P and BHIBA treated groups which likely underpin the lack of difference in weight between BHIBA treated mice and control (Supplementary Fig $6 p)$. BHIVA had no effect on the metabolic parameters independent of body mass.

MOVA, 5OP and BHIBA increased systemic energy expenditure in mice. We examined the expression of thermogenic and mitochondrial metabolism genes in BAT, subcutaneous inguinal WAT and skeletal muscle of the metabokine treated mice (Supplementary Fig $6 q-t$ ). Metabolite treatment also increased citrate synthase activity, a marker of mitochondrial density and TCA cycle flux, in the BAT, inguinal WAT and muscle of metabokine treated mice (Supplementary Fig $6 u-x$ ).

\section{MOVA, 5OP and BHIBA decrease weight gain, increase systemic energy expenditure and regulate glucose homeostasis in a mouse model of obesity and diabetes}

The candidate metabokines 5OP, MOVA and BHIBA increased energy expenditure and markers of oxidative metabolism in muscle and thermogenesis in adipose tissue in mice. Therefore, we investigated the effect of MOVA, 5OP, and BHIBA on the metabolic phenotype in a high fat feeding mouse model of obesity and T2DM. Six-week-old mice were treated with the metabokines (MOVA $100 \mathrm{mg} / \mathrm{kg} / \mathrm{day} ; 50 \mathrm{P}$ $100 \mathrm{mg} / \mathrm{kg} / \mathrm{day}$ and BHIBA $150 \mathrm{mg} / \mathrm{kg} /$ day) in drinking water for 17 weeks while fed a $60 \%$ fat diet. Plasma concentrations of the metabolites were significantly increased in treated mice (Supplementary Fig. $7 a-c)$. MOVA, 5OP and BHIBA significantly reduced weight gain in fat fed mice (Fig. 6a - c). Adiposity of the MOVA and 5OP treated mice was observed to be reduced by $17.1 \%$ and $19.4 \%$, 
respectively, using Computed Tomography (CT) (Fig. 6d). Consistent with the effect on adiposity and body weight, analysis with metabolic cages indicated that whole-body energy expenditure (Fig. $6 \mathrm{e}-\mathrm{g}$ ) and oxygen consumption (Supplementary Fig. 7d - f) were increased in the MOVA, 5OP and BHIBA treated high fat-fed mice, independent of body mass (as determined by ANCOVA). There was no significant difference in activity, food intake or water intake (Supplementary Fig. $7 g-i$ ).

Next, the mice were challenged with an insulin tolerance test (ITT) (Fig. $6 \mathrm{~h}-$ j) (Supplementary Fig. 7 j) and intraperitoneal glucose tolerance test (IPGTT) (Supplementary Fig. $7 \mathrm{k}-\mathrm{n}$ ). 5OP and BHIBA significantly improved both the insulin sensitivity and the glucose tolerance in the mice. MOVA treatment demonstrated a mild but significant improvement in insulin sensitivity.

MOVA, 5OP and BHIBA increased systemic energy expenditure and improved glucose homeostasis in mice. The metabolites also altered the metabolic phenotypes of adipocytes and skeletal myocytes in vitro and in chow fed mice in vivo. Therefore, we examined markers of thermogenesis and mitochondrial metabolism in BAT and subcutaneous WAT of the MOVA, 5OP and BHIBA treated mice. The activity of citrate synthase was significantly increased in the BAT of mice following MOVA, 5OP and BHIBA treatment, suggesting increased mitochondrial biogenesis (Fig 6k). Consistent with these data, IHC analysis of the BAT of MOVA, 5OP and BHIBA treated mice indicated increased concentrations of UCP1 (Supplementary Fig. 7o), which were confirmed by ELISA (Fig. 6I). PGC1a protein concentration was also increased in the BAT of BHIBA treated mice (Fig 6I). Citrate synthase activity was increased in subcutaneous WAT of mice following MOVA, 5OP and BHIBA treatment (Fig $6 \mathrm{~m}$ ). IHC analysis of inguinal subcutaneous WAT from these mice also indicated increased UCP1 concentrations following 5OP and BHIBA treatment (Supplementary Fig. 70), which were again confirmed by ELISA (Fig. 6n). 50P and BHIBA also increased the concentration of PGC1 a protein in inguinal WAT (Fig. 6n), with 5OP, BHIBA and MOVA all increasing CPT1 concentrations (Fig. 6n). MOVA and 5OP decreased adipocyte hypertrophy, significantly reducing adipocyte size within the inguinal WAT depot, consistent with effects of the metabolites on weight gain (Supplementary Figure. 7p).

MOVA, 5OP, and BHIBA, increased expression of mitochondrial and metabolic genes in skeletal myocytes in vitro and in vivo. Consequently we investigated markers of mitochondrial metabolism in the soleus muscle of the MOVA, 5OP and BHIBA treated murine model of obesity. Mitochondrial density was increased in skeletal muscle by all three metabolite signals (Fig. 60). Protein concentrations of PGC1a and NDUFS1 were significantly increased in the muscle of metabolite-treated mice (Fig. 6p).

\section{PET/CT imaging identifies increased glucose uptake into skeletal muscle and brown adipose tissue of metabolite treated mice}

Positron Emission Tomography / Computed Tomography (PET/CT) was used to determine the tissuespecific metabolic effects of MOVA, 5OP and BHIBA treatment in vivo in the mouse model of obesity and T2DM. PET/CT is commonly used to determine BAT location and activity ${ }^{21}$. Mice either treated with MOVA (100 mg/kg/day), 5OP (100 mg/kg/day) or BHIBA (150 mg/kg/day) were imaged using PET/CT 
and the glucose analogue ${ }^{18} \mathrm{~F}$-FDG (Fig. $\left.6 \mathrm{q}\right)$. The metabolic activity of BAT was significantly increased in BHIBA and MOVA treated mice (Fig. 6r). Hind limb skeletal muscle metabolic activity was increased in BHIBA, MOVA and 5OP treated mice (Fig. 6s), with forelimb muscle metabolic activity significantly increased in MOVA and 5OP treated mice (Fig. 6t). Together, these data show the metabokines increase energy expenditure, reduce weight gain, improve glucose homeostasis and increase glucose and fatty acid catabolism in BAT, WAT and skeletal muscle.

\section{MOVA and 50P have additive effects on body weight and glucose metabolism in mice}

Physiologically the candidate metabokines are generated in browning adipocytes and concomitantly increased in the plasma by stimulation of thermogenesis in mice. MOVA and 5OP produced the most robust and significant reduction in weight gain and adiposity in high fat fed mice and induced the greatest changes in metabolic phenotype in human adipocytes and myocytes. Therefore, we examined whether these metabolites would have combinatorial anti-obesity and anti-diabetic effects on systemic metabolism. Six-week-old mice were treated with a combination of MOVA (100 mg/kg/day) and 5OP (100 $\mathrm{mg} / \mathrm{kg} / \mathrm{day}$ ) in drinking water for 17 weeks and fed a $60 \%$ fat diet. The combination of metabolites additively reduced weight gain when compared to either 5OP or MOVA treatments alone (Supplementary Fig. 8a). CT analysis identified that a combination of MOVA and 5OP reduced body fat by $24.6 \%$ in treated mice compared with controls (Supplementary Fig. 8b). Glucose tolerance was further improved by a combination of 5OP and MOVA treatment (Supplementary Fig. 8c). PET/CT analysis using ${ }^{18} \mathrm{~F}-\mathrm{FDG}$ indicated that mice treated with both MOVA and 5OP had enhanced glucose uptake into the hind limb skeletal muscle when compared to the singly administered treatments (Supplementary Fig. 8d \& e). These data suggest MOVA and 5OP function through disparate mechanisms and that the small molecule adipokine-like signals function in concert to mediate systemic metabolism and anti-obesity effects on release from brown/beige adipose tissue.

\section{MOVA and 5OP signal through cAMP-PKA-p38 MAPK and BHIBA via mTOR to regulate adipocyte and myocyte metabolic gene expression}

We then determined whether the metabokines function extracellularly or intracellularly at the human adipocyte to induce expression of UCP1. MCTs can function to both import and export monocarboxylate species $^{22}$. Concomitant treatment of primary adipocytes with an MCT inhibitor (MCTi; a-cyano-4hydroxycinnamate) and 50P resulted in an abrogation of 5OP-induced UCP1 expression, suggesting that $50 P$ requires import into the adipocyte to induce molecular signals leading to increased UCP1 expression (Fig 7a). Conversely, inhibition of MCT activity did not impair MOVA or BHIBA-mediated UCP1 expression, with dual metabokine and MCT inhibitor treatment trending towards increased UCP1 expression compared to metabokine treatment alone (Fig $7 \mathrm{~b} \& \mathrm{c}$ ). These data suggest MOVA and BHIBA function through extracellular signal transduction and may require a receptor in the adipocyte membrane.

Canonical activation of adipocyte thermogenesis through $\beta_{3}$-adrenergic signaling requires intracellular signal transduction by cyclic AMP (CAMP) and downstream activation of Protein Kinase A (PKA) ${ }^{1}$. Using 
LC-MS we measured the intracellular cAMP content in human adipocytes treated with 50P, MOVA and BHIBA (Fig 7d). The concentration of CAMP was unchanged in BHIBA treated adipocytes but increased in 5OP and MOVA-treated cells. We then analyzed the cAMP content of BAT (Supplementary Fig 9a), subcutaneous WAT (Supplementary Fig 9b) and soleus skeletal muscle (Supplementary Fig 9c) of 50P, MOVA and BHIBA-treated mice. The CAMP content was increased in BAT, subcutaneous WAT and skeletal muscle of 5OP and MOVA-treated mice. We then co-treated primary adipocytes with either MOVA or 5OP and the selective PKA inhibitor H89. Inhibition of PKA impaired MOVA and 5OP-induced expression of brown adipocyte-associated genes in the adipocytes (Fig 7e \& $\mathrm{f}$ ) (Two-way ANOVA, $P<0.0001$ Control vs MOVA, $\mathrm{P}<0.0001$ MOVA vs MOVA + PKAi; $\mathrm{P}<0.0001$ Control vs 5OP, $\mathrm{P}=0.007$ 50P vs 5OP + PKAi).

The downstream signaling pathways induced by MOVA, 5OP and BHIBA in skeletal muscle and adipose tissue were investigated using phosphokinase profiling of BAT, subcutaneous WAT and soleus muscle from metabolite treated mice. Treatment with MOVA increased phosphorylation of members of the p38 mitogen-activated protein kinase family (p38 MAPK) in BAT (Supplementary Fig. 9d), subcutaneous WAT (Supplementary Fig. 9e) and soleus (Supplementary Fig. 9f). In soleus, phosphorylation of the p38 MAPK substrate glycogen synthase kinase 3 beta (GSK-3ß) was also increased by MOVA. In BAT, MOVA treatment increased phosphorylation of mitogen-activated protein kinase 3 (MKK3) located upstream of the p38 MAPKs in cellular signaling cascades. 50P was also observed to increase phosphorylation of p38 MAPKs in BAT (Supplementary Fig. 9g), subcutaneous WAT (Supplementary Fig. 9h) and soleus (Supplementary Fig. 9i). These findings are consistent with the requirement for p38MAPK in cAMPmediated expression of $\mathrm{UCP}{ }^{23}$. BHIBA increased phosphorylation of both the mammalian target of rapamycin (mTOR) and mTOR's downstream substrate p70S6 kinase (p70S6K) in BAT, subcutaneous WAT and soleus (Supplementary Fig. 9j - I) and increased the phospho mTOR / total mTOR ratio (Supplementary Fig 9m \& n). Inhibition of p38 MAPK signaling by a pan p38 MAPK inhibitor (500 nM BIRB 796) abrogated MOVA (Fig. 7g) and 5OP (Fig. 7h) induced metabolic gene expression (PPARa, CPT1b, ACADvl) in human primary skeletal myocytes (Two-way ANOVA, Control vs MOVA $P<0.01$, MOVA vs MOVA + p38 MAPKi $\mathrm{P}<0.0001$; Control vs 5OP $\mathrm{P}<0.0001$, 50P vs 5OP + p38 MAPKi $\mathrm{P}<0.0001$ ). Inhibition of mTOR (temsirolimus $500 \mathrm{nM}$ ) in skeletal myocytes impaired BHIBA-induced metabolic gene expression (Fig. 7i) (Two-way ANOVA, Control vs BHIBA P < 0.01, MOVA vs MOVA + mTORi $P<0.01$ ). In human primary adipocytes, the inhibition of p38 MAPK signaling reduced MOVA and 50P-induced brown adipocyte associated gene expression (Fig. 7j \& 7k) (Two-way ANOVA, Control vs MOVA $P<0.0001$, MOVA vs MOVA + p38MAPKi $P<0.01$, Control vs 50P $P<0.0001$, 50P vs 50P + p38MAPKi $P<0.0001$ ). Inhibition of mTOR signaling with temsirolimus reduced BHIBA-induced expression of brown adipocyteassociated genes in human primary adipocytes (Fig. 7l) (Two-way ANOVA, Control vs BHIBA $P<0.0001$, BHIBA vs BHIBA + mTORi $\mathrm{P}<0.0001)$.

These data suggest that MOVA and 5OP function differentially via extracellular and intracellular mechanisms, respectively, to induce metabolic reprogramming in skeletal myocytes and adipocytes via a cAMP-PKA-p38 MAPK-mediated signaling pathway. BHIBA induces the thermogenic genes in adipocytes 
and mitochondrial gene expression in myocytes through extracellular activation of downstream mTOR signaling.

\section{Concentrations of the adipokinelike metabolites in adipose tissue and plasma are inversely correlated with body mass index in humans}

We investigated the association between genetic variants in the genes encoding the metabokine biosynthetic enzymes and body mass index (BMI) in a large-scale Genome Wide Association Study (GWAS) database in Genetic Investigation of ANthropometric Traits (GIANT) and UK Biobank Metaanalysis ${ }^{24}$, included in the 795,640 subjects in the Type 2 Diabetes Knowledge Portal (http://www.type2diabetesgenetics.org/). We found that common noncoding variants in the MOVA, BHIBA and BHIVA biosynthetic genes (BCAT2, BCKDHB, ACADS and HADHA), the 5OP biosynthetic genes (GSS GGCT1) and the gene for MCT1 were significantly associated with BMI (Supplementary Table 6). The most significant variants in each gene for BMI were: BCKDHB rs13220420, $\mathrm{P}=0.00000750$; BCAT2 rs73587808, $P=0.000488 ;$ ACADS rs12369156, $P=0.000131 ;$ HADHA rs559393527, $P=0.0000341 ; G S S$ rs2236270, $\mathrm{P}=3.60 \mathrm{e}-8 ;$ GGCT rs549124813, $\mathrm{P}=0.0000875$ and MCT1/SLC16A1 rs186286251, $\mathrm{P}=$ $0.000471)$.

We then examined the association of subcutaneous WAT MOVA, 5OP, BHIVA and BHIBA concentrations with BMI in human volunteers (Supplementary Table 7). The WAT concentration of MOVA, 5OP and BHIVA were significantly inversely correlated with BMI (Fig. 8 a - d). Plasma concentrations of the metabolite adipokine-like signals were also inversely correlated with BMI (Supplementary Fig. $10 \mathrm{a}-\mathrm{d}$ ).

The association of metabokine concentrations with beige adipose tissue in humans was also interrogated. RNA was isolated from the adipose tissue of 30 volunteers and the expression of UCP1 and $C P T 1 b$ measured using RT-qPCR. Associations between the adipose tissue metabolite concentrations and the expression of $U C P 1(F i g ~ 8 \mathrm{e}-\mathrm{h}$ ) and $C P T 1 b$ (Fig. $8 \mathrm{i}-\mathrm{I})$ were analyzed. Concentrations of MOVA, 5OP and BHIVA were significantly correlated with tissue expression of UCP1 and CPT1b.

These data suggest that the metabokines are functionally associated with human physiology and may influence body mass phenotypes.

\section{Discussion}

The canonical role of BAT, and to some extent beige adipose tissue, has long been regarded as to generate heat through uncoupled respiration. However, BAT and beige adipose tissue may have a more varied capacity to regulate systemic metabolism. The ability of WAT to function as an endocrine organ, releasing messengers known as adipokines that coordinate a systemic response to energetic status, feeding behaviors and inflammatory responses, amongst other physiological processes, is well established $^{25}$. A similar endocrine function of BAT and beige adipose tissue remains poorly understood and characterized. However, evidence suggests the presence of endocrine and paracrine signals emanating from BAT and beige adipose tissue. Transplantation studies of both beige and brown fat in 
mice have demonstrated the capacity to induce weight loss in mouse models of obesity. Surprisingly, these studies have identified direct improvements in glucose homeostasis in skeletal muscle and activation of endogenous beige and brown fat ${ }^{8,9,26}$. In addition, the total loss of BAT has a more profound effect on metabolic status than the tissue specific ablation of UCP1. The ability of BAT to influence systemic energy balance is not solely reliant on non-shivering thermogenesis ${ }^{12-15}$.

Recent discoveries have highlighted a number of peptidic, lipid and miRNA brown adipokines ${ }^{27}$, including the discovery by Kong and colleagues of a BAT-to-muscle signaling axis mediated through the protein myostatin ${ }^{10}$. We have previously highlighted a small molecule metabolite-mediated skeletal muscle to beige adipose tissue signaling axis ${ }^{28}$. Here we identify MOVA, 5OP and BHIBA as a discrete set of unique, first in their class, metabolite brown and beige adipose metabokines. The effects of MOVA, 5OP and BHIBA in vitro and in vivo have been summarized and compared in Supplementary Table 8 . These metabolites function in concert to mediate crosstalk between BAT, beige adipose tissue and skeletal muscle by inducing expression of key mitochondrial genes and an oxidative phenotype in adipose and muscle, increasing whole-body energy expenditure, complementary to BATokine proteins and lipokines. To our knowledge these molecules represent the first examples of metabokines released from browning adipose which may then regulate muscle metabolism.

MOVA, BHIBA and BHIVA are monocarboxylic acids generated via the catabolism of BCAAs. Catabolites of BCAAs are emerging as novel bioactive metabolites and have been implicated in endocrine signaling. Notably, the valine catabolite $\beta$-aminoisobutyric acid (BAIBA) acts as an exercise-stimulated myokine, signaling to induce browning of WAT and hepatic fatty acid oxidation ${ }^{28}$. BHIBA is also a product of valine metabolism and signals between skeletal muscle and the endothelium in a PGC1a-dependent manner to increase fatty acid uptake ${ }^{29}$. Increased circulating plasma concentrations of BCAAs are a distinguishing feature of obesity and may predict T2DM onset ${ }^{30-34}$. Indeed, BCAAs can regulate protein synthesis and degradation, insulin secretion, and energy balance ${ }^{35}$. However, adipose tissue is capable of substantial BCAA catabolism and can modulate circulating BCAA concentrations ${ }^{36}$. The rate of BCAA catabolism is particularly high in BAT and can regulate whole-body energy homeostasis ${ }^{37-39}$. BCAA catabolic enzymes are downregulated in adipose tissue both in obesity and in insulin resistence ${ }^{38,40}$. Here, we identify increased BCAA degradation to generate MOVA, BHIBA and BHIVA in browning adipose tissue, a positive correlation between the subcutaneous adipose tissue concentration of these BCAA catabolites and brown adipocyte-associated gene expression in humans, and an inverse correlation between the adipose tissue concentration of the metabolites and BMI. We also describe non-coding variants in the metabolite biosynthetic genes which are significantly associated with BMI in large population GWAS meta-analysis. There is a loss of beige and brown adipose tissue in the so called "whitening" effect associated with obesity ${ }^{41}$. In addition, the disruption of BCAA catabolism in BAT impairs thermogenesis and reduces tissue UCP $1{ }^{42,43}$. Our work provides a link between the dysregulation of BCAA catabolism in adipose tissue, whitening of adipose depots, increased circulating BCAAs, and metabolic diseases including obesity. Metabolic risk associated with increased circulating BCAAs may, in part, be mediated by 
decreased biosynthesis and secretion of these metabolite brown and beige adipokines and perturbation of the inter-organ signaling axes they mediate.

$50 P$ is a neglected amino acid which links glutamine metabolism to glutathione biosynthesis. Glutathione is a potent antioxidant and protects against adverse cellular redox states. Browning of adipose tissue has been postulated as an adaptive mechanism to alleviate redox pressure ${ }^{44}$, with recent studies demonstrating that the expression of UCP1 is regulated by redox status including oxidative stress and antioxidants ${ }^{45,46}$. Thus, our identification of 50P as a brown and beige adipokine may represent a mechanism through which beige/brown adipose can communicate redox state and, amongst other effects, recruit additional beige adipocytes to rescue systemic redox stress.

We acknowledge limitations to our study. Our work does not preclude the presence of other metabolite factors, protein signals, or bioactive lipids that may contribute to the functional signaling between adipose-adipose and adipose-skeletal muscle. Indeed, our data suggests the presence of an, as yet unidentified, secreted peptidic inhibitor of browning. Due to the importance of the biosynthetic enzymes involved in the generation of BHIBA, BHIVA, 5OP and MOVA, shared across multiple metabolic pathways including BCAA catabolism, fatty acid oxidation, TCA cycle, glutamine/glutamate and redox glutathione metabolism, directly targeting these in loss-of-function experiments would be incapable of unambiguously distinguishing the effect of the metabolites from perturbations of multiple pathways. However, this observation supports our finding that these metabolites function as key brown and beige adipokines, as the need to closely integrate signals influencing systemic energy balance with pathways responding to cellular energy and redox metabolism would be essential. Our analyses suggest that MOVA and 5OP function through cAMP-PKA-p38 MAPK signaling, and BHIBA functions through mTOR, to induce adipocyte and myocyte metabolic gene expression. Both cAMP-PKA-p38 MAPK and mTOR signaling regulate the activation of BAT thermogenesis, WAT browning and mitochondrial biogenesis 23,47-49. Our study suggests that BHIBA and MOVA function through extracellular receptors and that 5OP induces effects through a direct metabolic mechanism to induce adipocyte browning. Future work may also uncover the effects of BHIBA, 50P and MOVA on other tissues, and the identity of the discreet receptors through which the metabokines exert their effects.

In addition, we ascertained that the metabolite BHIVA demonstrated some capacity to regulate adipose tissue metabolism in vitro, although no effect on energy or glucose homeostasis was observed in vivo. BHIVA has been shown to have bioactive properties in other settings, including skeletal muscle protein synthesis and exercise ${ }^{50}$, and our work does not preclude an alternative signaling role for this metabolite linking beige adipose tissue and BAT to the regulation of systemic physiology.

We identify MOVA, 5OP, and BHIBA as a unique and discrete set of small molecule brown and beige adipose metabokines. The identification of these metabolites as inter-organ signals has significant implications, not only for our understanding of the integration and regulation of physiological energy metabolism and its protective role against the development of metabolic diseases, but also for 
understanding the pathophysiology of, and potential therapeutics for obesity, T2DM and the metabolic syndrome.

\section{Online Methods}

\section{Culture and differentiation of mouse primary adipocytes}

Primary white adipose stromal vascular cells were fractionated from 6-10-week-old C57BL/6J male mice as previously described ${ }^{28,51}$. Stromal vascular cells were then cultured and differentiated into adipocytes according to published methods ${ }^{28,52}$. During the 6-day differentiation, cells were cultured with $100 \mathrm{nM}$ GW0742 (Sigma Aldrich), 250 nM Hydroxyisocaproic acid (HIC) (Sigma Aldrich), $10 \mu M$ a-ketoisovaleric acid (AKV) (Sigma Aldrich), $5 \mu \mathrm{M}$ a-hydroxyisovaleric acid (AHI) (Sigma Aldrich), $20 \mu \mathrm{M}$ 3-methyl-2oxovaleric acid (MOVA) (Sigma Aldrich), $20 \mu \mathrm{M}$ 5-oxoproline (5OP) (Sigma Aldrich), $20 \mu \mathrm{M} \beta$ hydroxyisobutyric acid (BHIBA) (Sigma Aldrich) and $10 \mu \mathrm{M} \beta$-hydroxyisovaleric acid (BHIVA) (Sigma Aldrich) or with $1 \mu \mathrm{M}$ forskolin (Sigma Aldrich) on days 5 - 6 of differentiation.

For media transfer experiments, treatment media was removed, cells were washed three times with phosphate buffered saline (PBS), and $1.1 \mathrm{ml}$ serum-free media was conditioned on fully differentiated primary white adipocytes that had remained untreated (control) or had received either 100 nM GW0742 throughout differentiation or $1 \mu \mathrm{M}$ forskolin on days 5 - 6 of differentiation within each well of a 12 well plate. Media was boiled at $100^{\circ} \mathrm{C}$ for 5 mins. Conditioned media ( $1 \mathrm{ml}$ ) was added to naïve fully differentiated primary adipocytes in each well of a 12 well plate. For assessment of aqueous fraction bioactivity, aqueous soluble metabolites were extracted from $1 \mathrm{ml}$ of conditioned media as previously described $^{53}$. Dried aqueous fractions were then reconstituted in $1 \mathrm{ml}$ of fresh serum-free media by vortexmixing ( $5 \mathrm{~min}$ ) followed by sonication ( $15 \mathrm{~min}$ ) and then added to naïve primary adipocytes in a 12 well plate for $24 \mathrm{hrs}$.

Mouse primary brown preadipocytes were isolated from C57BL6/J mice, cultured and differentiated according to established protocols ${ }^{54}$.

\section{Human primary adipocyte culture}

Human white primary preadipocytes (PromoCell, Heidelberg, Germany) were seeded (10,000-15,000 cells $\left./ \mathrm{cm}^{2}\right)$ and grown to confluence $\left(37^{\circ} \mathrm{C}, 5 \% \mathrm{CO}_{2}\right)$ in preadipocyte growth medium $(0.05 \mathrm{ml} / \mathrm{ml}$ fetal calf serum, $0.004 \mathrm{ml} / \mathrm{ml}$ endothelial cell growth supplement, $10 \mathrm{ng} / \mathrm{ml}$ epidermal growth factor, $1 \mu \mathrm{g} / \mathrm{ml}$ hydrocortisone, $90 \mu \mathrm{g} / \mathrm{ml}$ heparin). Preadipocytes were differentiated according to supplier's instructions. Briefly, growth medium was replaced by differentiation media $(8 \mu \mathrm{g} / \mathrm{ml}$ d-biotin, $0.5 \mu \mathrm{g} / \mathrm{ml}$ insulin, $400 \mathrm{ng} / \mathrm{ml}$ dexamethasone, $44 \mu \mathrm{g} / \mathrm{ml}$ isobutylmethylxanthine, $9 \mathrm{ng} / \mathrm{ml}$ L-thyroxine, $3 \mu \mathrm{g} / \mathrm{ml}$ ciglitazone) for 48 hours. Differentiation medium was replaced with adipocyte nutrition media $(0.03 \mathrm{ml} / \mathrm{ml}$ fetal calf serum, $8 \mu \mathrm{g} / \mathrm{ml}$ d-biotin, $0.5 \mu \mathrm{g} / \mathrm{ml}$ insulin, $400 \mathrm{ng} / \mathrm{ml}$ dexamethasone) for 12 days until cells were fully differentiated. Media was changed every 48 hours. 
Human white primary adipocytes were treated throughout differentiation with either $100 \mathrm{nM}$ GW0742, 3methyl-2-oxovaleric acid (MOVA) $(10 \mu \mathrm{M}, 20 \mu \mathrm{M}, 40 \mu \mathrm{M})$, 5-oxoproline (5OP) $(10 \mu \mathrm{M}, 20 \mu \mathrm{M}, 40 \mu \mathrm{M}), \beta$ hydroxyisobutyric acid (BHIBA) $(10 \mu \mathrm{M}, 20 \mu \mathrm{M}, 40 \mu \mathrm{M})$ and $\beta$-hydroxyisovaleric acid (BHIVA) $(5 \mu \mathrm{M}, 10$ $\mu \mathrm{M})$, with $1 \mu \mathrm{M}$ forskolin on days 10 - 12 of differentiation and with either $2 \mathrm{mM}$ a-cyano-4-

hydroxycinnamate for $24 \mathrm{hrs}$ on day 11 of differentiation, or $500 \mathrm{nM}$ of the p38 MAPK inhibitor Birb 796, $500 \mathrm{nM}$ of the mTOR inhibitor temsirolimus, or $10 \mu \mathrm{M}$ of the PKA inhibitor H89 for 12 hours on day 12 of differentiation. All experiments were performed using adipocytes (day 12) at passages $3-5$.

\section{C2C12 myocytes}

C2C12 myocytes were cultured as previously described ${ }^{55}$.

\section{Human primary skeletal myocyte culture}

Adult human skeletal myoblasts (Cell applications Inc.) were grown in human skeletal muscle cell growth medium (Cell applications Inc.) at $37^{\circ} \mathrm{C}$ within a humidified atmosphere at $5 \% \mathrm{CO}_{2}$. Subculture of human skeletal myoblasts occurred once $85 \%-95 \%$ confluency was reached. Experiments were limited to the $5^{\text {th }}$ passage. Human skeletal myoblasts were seeded at 9,500 cells per $\mathrm{cm}^{2}$. Once confluent, myoblasts were cultured for $6-8$ days in skeletal muscle differentiation media (Cell applications Inc.) to induce myoblast differentiation to myotubes. Myoblasts were treated with human skeletal muscle cell differentiation medium (Cell applications Inc.) containing either BHIBA $(5 \mu \mathrm{M}$ and $20 \mu \mathrm{M})$, MOVA $(5 \mu \mathrm{M}$ and $20 \mu \mathrm{M}), 50 \mathrm{P}$ $(5 \mu \mathrm{M}$ and $20 \mu \mathrm{M}), \mathrm{BHIVA}(2.5 \mu \mathrm{M}$ and $10 \mu \mathrm{M})$, p38 MAPK inhibitor Birb 796 (12 hours on Day 8, $500 \mathrm{nM})$ (Sigma) or temsirolimus (12 hours on Day $8,500 \mathrm{nM}$ ).

\section{Immortalized human white preadipocyte culture}

Immortalized human white preadipocytes, isolated from human neck fat, were obtained from Yu-Hua Tseng (Joslin Diabetes Center, Harvard Medical School, USA) ${ }^{56}$. Cells were cultured according to a previously published protocol ${ }^{56}$. Cells were treated throughout induction and maintenance phases with either $20 \mu \mathrm{M}$ MOVA, $20 \mu \mathrm{M}$ 5OP, $20 \mu \mathrm{M}$ BHIBA or $10 \mu \mathrm{M}$ BHIVA or for the final 4 days of differentiation with $1 \mu \mathrm{M}$ forskolin.

\section{Gene expression array}

Total RNA was extracted and reverse transcribed into cDNA using ambion TaqMan Gene Expression Cells-to- $C_{\mathrm{T}}$ kits (AM1729). cDNA was preamplified using TaqMan PreAmp MasterMix Kits (Applied Biosystems 4384267), prior to loading on TaqMan OpenArray Real-Time PCR Plates (Applied Biosystems 4406947) and analysis by RT-PCR on a QuantStudio 12K Flex RT-PCR System.

Data analysis was carried out in the Thermo Fisher Cloud Relative Quantification App (3.4.1-PCR-build3 2017-09-26). Data was normalized to PPIA, RPLP0 and B2M.

\section{Confocal images and image quantification}


Cells were washed with PBS and labelled with 1:500 HCS Lipidtox Green (ThermoFisher, H34475) and and 1:10000 Hoechst 33342 (ThermoFisher, $\mathrm{H} 3570$ ) at $37^{\circ} \mathrm{C}$ for 30 minutes. Afterwards, cells were washed three times with PBS, fixed in $3.7 \%$ formaldehyde for 15 minutes, and washed four times with PBS. Cells were resuspended in donkey blocking buffer (1xPBS, $5 \%$ donkey serum, $0.3 \%$ Triton X-100) and incubated at $4^{\circ} \mathrm{C}$ overnight. Blocking buffer was removed prior to addition of mouse anti-human/antimouse UCP-1 monoclonal antibody (R\&D Systems MAB6158, Minneapolis, USA) in antibody buffer $\left(10 \mu \mathrm{g} / \mathrm{ml}, 1 \times P B S, 1 \%\right.$ BSA, $0.3 \%$ Triton X-100) for one hour at $21^{\circ} \mathrm{C}$. Cells were washed three times with PBS, Alexa455 AffiniPure donkey anti-mouse IgG $(\mathrm{H}+\mathrm{L})$ antibody (Jackson ImmunoResearch, West Grove, USA) in antibody buffer $\left(3 \mu \mathrm{g} / \mathrm{ml}\right.$ in $1 \times$ PBS, $1 \% \mathrm{BSA}, 0.3 \%$ Triton X-100) was added for one hour at $21^{\circ} \mathrm{C}$ and washed three times with PBS. Samples were afterwards kept in PBS.

Imaging was performed on an OPERA QEHS spinning disc confocal microscope (PerkinElmer) using an X20-magnification water immersion lens (numerical aperture 0.7 ), and $405 \mathrm{~nm}, 488 \mathrm{~nm}$, and $561 \mathrm{~nm}$ lasers with appropriate filter sets. Image analysis was performed using Columbus software (PerkinElmer). To determine UCP1 protein levels, firstly areas with differentiated adipocytes were defined using LipidTox labelling of lipid droplets followed by measurement of mean UCP1 intensity levels within this region.

\section{Fatty acid and glucose uptake assays}

The cellular fatty acid uptake assay was performed as described in the literature ${ }^{29}$.

The cellular glucose uptake assay was performed as follows. Cells were grown and differentiated in 96well plates. Cells were washed with Dulbecco's phosphate buffered saline (DPBS) and placed in low glucose ( $1 \mathrm{~g} / \mathrm{L})$ serum-free Dulbecco Modified Eagle Medium (DMEM) for 24 hours. Media was aspirated and replaced with fresh low glucose serum-free DMEM for 1 hour. Following media aspiration DPBS containing 6-deoxy-6-[(7-nitro-2,1,3-benzoxadiazol-4-yl)amino]-D-glucose (6-NBDG) (200 $\mu \mathrm{M})$ was added for 1 hour and cells were kept at $37^{\circ} \mathrm{C}, 5 \% \mathrm{CO}_{2}$. Cells were washed three times with DPBS and fluorescence measured using a microplate reader (excitation $485 \mathrm{~nm}$, emission $528 \mathrm{~nm}$ ).

\section{${ }^{13} \mathrm{C}$-Palmitate substrate labeling study}

Palmitate was solubilized using a dialyzed albumin solution ${ }^{53} \cdot{ }^{13} \mathrm{C}$-Palmitate labeling studies were performed as previously described ${ }^{52}$. Briefly, fully differentiated human primary white adipocytes were conditioned with serum-free medium containing insulin $850 \mathrm{nmol} / \mathrm{L}$, triiodothyronine $1 \mathrm{nmol} / \mathrm{L}$, and rosiglitazone $1 \mu \mathrm{mol} / \mathrm{L}$ and $140 \mu \mathrm{mol} / \mathrm{L} \mathrm{U}-{ }^{13} \mathrm{C}$-labeled palmitate (Cambridge Isotope Laboratories). After 24 hours, cells were collected and metabolites extracted as previously described. During differentiation, cells were cultured with either $20 \mu \mathrm{M}$ MOVA, $20 \mu \mathrm{M}$ 5OP, $20 \mu \mathrm{M}$ BHIBA or $10 \mu \mathrm{M}$ BHIVA.

\section{${ }^{13} \mathrm{C}$-labeled amino acid substrate analysis}

Fully differentiated human primary white adipocytes were conditioned with serum-free medium containing insulin $850 \mathrm{nmol} / \mathrm{L}$, triiodothyronine $1 \mathrm{nmol} / \mathrm{L}$, and rosiglitazone $1 \mu \mathrm{mol} / \mathrm{L}$ and either 200 
$\mu \mathrm{mol} / \mathrm{L} \mathrm{U}{ }^{13} \mathrm{C}$-labeled leucine, $200 \mu \mathrm{mol} / \mathrm{L} \mathrm{U}-{ }^{13} \mathrm{C}$-labeled isoleucine, $200 \mu \mathrm{mol} / \mathrm{L} \mathrm{U}-{ }^{13} \mathrm{C}$-labeled valine or $100 \mu \mathrm{mol} / \mathrm{L} \mathrm{U}-{ }^{13} \mathrm{C}$-labeled glutamate (Cambridge Isotope Laboratories). After 24 hours, cells were collected and metabolites extracted as previously described. During differentiation, cells were cultured with $1 \mu \mathrm{M}$ forskolin on days $10-12$ of differentiation. Extracted metabolites were analyzed by GC-MS as previously described ${ }^{52,53}$.

\section{Animal experimentation}

Six-week-old C57BL6/J mice (Charles River) were weight matched and assigned to groups for treatment). Mice were treated with either $100 \mathrm{mg} / \mathrm{kg} /$ day 5OP, $100 \mathrm{mg} / \mathrm{kg} /$ day MOVA, $150 \mathrm{mg} / \mathrm{kg} /$ day BHIBA or 125 $\mathrm{mg} / \mathrm{kg} /$ day BHIVA in their drinking water for 17 weeks and fed either standard chow ( $\mathrm{n}=5$ per group) or a $60 \%$ fat diet ad libitum (Bio Serv F3282) ( $n=10$ per group). Animals were housed in conventional cages at room temperature with a 12 hour light/dark photoperiod.

The cold exposure study was conducted at the University of Cambridge. Animals were housed in a specific pathogen free facility with 12 hour light and 12 hour dark cycles. Four groups of $8 \mathrm{C} 57 \mathrm{BL} 6 / \mathrm{J}$ mice (Charles River) underwent thermal adaptation at 12 weeks of age. One group was placed at $8^{\circ} \mathrm{C}$ for 4 weeks, a second group was maintained at room temperature for 3 weeks then placed at $8^{\circ} \mathrm{C}$ for one week, a further group was placed at $28^{\circ} \mathrm{C}$ for 4 weeks and a final group was maintained at room temperature for 4 weeks $\left(21-23^{\circ} \mathrm{C}\right)$. All mice were killed at 16 weeks of age. This study was regulated under the Animals (Scientific Procedures) Act 1986 Amendment Regulations 2012 following ethical review by the University of Cambridge Animal Welfare and Ethical Review Body (AWERB). All procedures were carried out in accordance with U.K. Home Office protocols by a personal license holder.

\section{Indirect calorimetry}

All experiments were performed according to previously published protocols ${ }^{28}$. Briefly Combined Laboratory Animal Monitoring System (CLAMS) (Columbus Instruments) was used to monitor oxygen consumption, carbon dioxide production, food intake, and locomotory activity. The CLAMS was calibrated before each experiment. Animals were subjected to a 3-day acclimation period in a training cage to habituate to the environment of the metabolic cages. Animals were maintained in normal bedding at $22^{\circ} \mathrm{C}$ throughout the monitoring period. Ten minute interval measurements for each animal were obtained for oxygen and carbon dioxide with ad libitum access to food and water (or water plus metabolites) on a controlled 12 hour light/dark cycle. Cages contained one mass sensor to monitor food intake. Data were analyzed using CaIR ${ }^{57}$.

\section{Intraperitoneal glucose tolerance tests}

Intraperitoneal glucose tolerance tests were performed as previously described ${ }^{28}$.

\section{Positron Emission Tomography / Computed Tomography}


PET/CT scans were performed on an Albira Si (Bruker). Mice were anaesthetized under 2-3\% isoflurane, weighed and injected intravenously with $8.9 \mathrm{MBq} \pm 2.3 \mathrm{MBq} 18 \mathrm{~F}$ Fluorodeoxyglucose ( $\left.{ }^{18} \mathrm{~F}-\mathrm{FDG}\right)$ in $200 \mu \mathrm{L}$ via the lateral tail vein and flushed with saline, followed by a 1 hour uptake period. Mice were scanned for 20 minutes static PET, followed by a 10 minute CT protocol for anatomical registration. The CT scans were performed at a $35 \mathrm{kV}$ tube voltage and $200 \mu \mathrm{A}$ over 250 projections. Animal temperature was maintained and monitored throughout the procedure alongside the respiratory rate. PET/CT data was reconstructed using the Albira Reconstructor Software (Bruker). PET data was reconstructed using a maximum likelihood expectation maximization (MLEM) iterative method at 25 iterations with scatter, random event and radiotracer decay corrections. The PET data was fused with the CT data which was reconstructed with filtered back projection (FBP). PET and CT image data was analyzed in PMOD, version 3.807. The methods used to calculate adiposity have been described previously ${ }^{58}$.

\section{Blood and Tissue Collection}

Mice were killed by cervical dislocation. Blood was obtained by cardiac puncture, collected in tubes containing EDTA ( $2.5 \mathrm{mmol} / \mathrm{L}$ ) and immediately centrifuged to obtain plasma. WAT, interscapular BAT, soleus and gastrocnemius muscle were removed and flash frozen in liquid nitrogen.

\section{Gene Expression Analysis}

Total RNA extraction from WAT, adipocytes and myocytes; cDNA conversion; and quantitative RT-PCR were performed according to published protocols ${ }^{28}$. All data were normalized to $18 \mathrm{~S}$ rRNA (human WAT, adipocytes and myocytes) and quantitative measures were obtained using the $\Delta \Delta C T$ method.

\section{RNA-Seq}

Next-generation RNA sequencing was performed by Cambridge Genomic Services (Cambridge UK). The Lexogen 3'mRNA seq kit (illumina) was used for library preparation. Samples were analyzed using a NextSeq500 (illumina) with 75 bp per read and 10 million reads per sample. RNA-Seq data associated with this study is available from Gene Expression Omnibus [GSE129153].

\section{Protein Analysis}

Analysis of UCP-1, PGC-1a, CPT1 and NDUFS1 was performed using ELISA per the manufacturer's instructions (UCP1 Kit SEF557Ra, PGC-1a Kit SEH337Ra; CPT1 SEF360Mu; NDUFS1 SEJ794Hu CloudClone Corp., Houston, TX). Kinase profiling was performed using the Proteome Profiler Phospho-MAPK Array Kit (Bio-techne Ltd; ARY002B) according to the manufacturer's instructions.

\section{Citrate Synthase Assay}

Citrate synthase was assayed according to published protocols ${ }^{52}$.

\section{Histology and Immunohistochemistry}


Tissue was fixed in $4 \%$ paraformaldehyde, processed in paraffin and sectioned into $4 \mu \mathrm{m}$ sections for staining. Sections were deparaffinized in xylene, rehydrated through a $95-50 \%$ ethanol series, then placed in water before staining. The VECTASTAIN Elite ABC HRP Kit (Vector Labs, cat no: PK 6100) was used as per manufacturer's instructions to retrieve antigens. Anti-UCP1 primary antibody (abcam ab23841) was used at a 1:100 dilution and incubated on sections in a humidity chamber at room temperature for 60 minutes. Visualization of VECTASTAIN peroxidases was achieved using VECTOR NovaRED Peroxidase (HRP) Substrate Kit (Vector Labs, cat no: SK-4800). Sections were rinsed in tap water and counterstained with hematoxylin (VECTOR Hematoxylin QS, Vector Labs, Cat no: H-3404). Sections were rinsed in tap water, dehydrated through a 50-95\% ethanol series, cleared in xylene and mounted onto coverslips using DPX. Pictures were captured using a standard light microscope and ZEISS Zen 2 pro software.

\section{Respirometry}

Basal respiration was measured in adipocytes and skeletal myocytes maintained in Krebs-Henseleit buffer at $37^{\circ} \mathrm{C}$ using Clark-type oxygen electrodes (Strathkelvin Instruments, Strathkelvin, U.K.) as described previously ${ }^{52}$. In addition, respiration was also measured in human primary adipocytes following the addition of succinate $(20 \mathrm{mM})$.

Mitochondrial respiration profiles of immortalized human white preadipocytes isolated from neck fat and differentiated to mature adipocytes in the presence of MOVA $(20 \mu \mathrm{M}), 50 \mathrm{P}(20 \mu \mathrm{M})$, BHIBA $(20 \mu \mathrm{M})$, or BHIVA $(10 \mu \mathrm{M})$ were assessed on a Seahorse XFe96 Flux Analyzer (Agilent Technologies, Waldbronn, Germany), using Agilent MitoStress kits according to manufacturer's instructions. Briefly, immortalized human white preadipocytes were seeded into XFe96 cell culture plates and cultured as described earlier. Oxygen consumption rates (OCR) were monitored during the sequential injection of oligomycin $(1 \mu \mathrm{M}$, ATP-Synthase coupled respiration), carbonyl-cyanide-4-(trifluoromethoxy)-phenylhydrazone ( $1 \mu \mathrm{M}$, maximal respiration) and rotenone/antimycin $\mathrm{A}(0.5 \mu \mathrm{M}$ each, non-mitochondrial respiration). OCR were afterwards normalized to cell number as determined by Hoechst 33342 staining and measurement on a Cytation 5 (BioTek, Winooski, USA) with DAPI filter.

High-resolution respirometry was carried out on human primary skeletal myoblasts differentiated to mature myotubes in the presence of MOVA $(20 \mu \mathrm{M})$ or 5OP $(20 \mu \mathrm{M})$ using an Oxygraph $2 \mathrm{k}$ (Oroboros, Innsbruck). Cells were suspended in $2.4 \mathrm{~mL}$ MiR05 (MiR05: $110 \mathrm{mM}$ sucrose, $60 \mathrm{mM}$ K-lactobionate, 20 mM HEPES, $20 \mathrm{mM}$ taurine, $10 \mathrm{mM} \mathrm{KH2PO4,} 3 \mathrm{mM} \mathrm{MgCl} 2,0.5 \mathrm{mM} \mathrm{EGTA}, 1 \%$ (w/v) fatty-acid free BSA, $\mathrm{pH}$ 7.1). $100 \mu \mathrm{L}$ of cell suspension was used to determine cell counts using a Millipore Scepter Handheld Automated Cell Counter. Respiration rates of myocytes were measured using Clark-type polarographic oxygen electrodes (Oxygraph-2k; Oroboros, Innsbruck) maintained at $37^{\circ} \mathrm{C}$ under a constant stir speed of $600 \mathrm{rpm}$. Cells were permeabilized with digitonin (Caymann Chemicals, $2.5 \mu \mathrm{g} / \mathrm{mL}$ ) immediately prior to adding the suspension into the Oxygraph chamber. Substrates were added to the chamber and steady rates of respiration recorded. Substrates were added to the chamber in the following order giving the final concentrations in the chamber indicated; Glutamate 10 mM; Malate 0.5 mM; 0.5 mM; ADP; Octanoyl- 
Carnitine (APExBIO Technology); 2.5 mM; Cytochrome c $10 \mu \mathrm{M}$; Succinate 10 mM; FCCP $5 \mu$ M; Antimycin A $12.5 \mu \mathrm{M}$. Respiration rates were corrected for cell number.

\section{Tissue and Primary Cell Metabolite Extraction}

Metabolites were extracted from WAT, BAT, skeletal muscle and primary skeletal myocytes and adipocytes as previously described ${ }^{53}$.

\section{GC-MS Metabolomic Analysis}

Dried aqueous and organic phase samples were derivatized using the method described previously ${ }^{53}$. GCMS metabolomics and data analysis were performed according to published methods ${ }^{53}$.

\section{LC-MS Metabolomic Analysis}

LC-MS metabolomics analysis was performed using a 4000 QTRAP triple quadrupole mass spectrometer (Applied Biosystems/Sciex) coupled to an Acquity UPLC according to a previously described method $^{28,52,59}$. cAMP was measured as previously described ${ }^{52}$. All metabolite concentrations were determined using the standard addition method ${ }^{28}$.

\section{Human Adipose Biopsies}

Forty-two eligible and consecutive patients undergoing routine de-novo pacemaker implantation at Leeds General Infirmary volunteered to participate in the study and provided written consent (Supplementary Table 7). Human subcutaneous adipose tissue biopsies were obtained prior to any instrumentation of the central circulation. Lidocaine was initially injected to anaesthetize the area and a small incision was made under the left clavicle. Immediately prior to creation of the pre-pectoral pocket for the generator above the pectoralis major a portion of subcutaneous adipose tissue (approximately 100mg) was sampled. This sample was immediately snap frozen in liquid nitrogen. Following this, pacemaker procedures were otherwise completed routinely. There were no complications ascribed to the sampling procedure. The study is approved by the Leeds West Research Ethics Committee (11/YH/0291) and Leeds Teaching Hospitals Trust R\&D committee (CD11/10015) and conforms to the Declaration of Helsinki.

\section{Human GWAS database analysis}

The Type 2 Diabetes Knowledge Portal (http://www.type2diabetesgenetics.org/) was searched for genetic variants in the genes encoding the metabokine biosynthetic enzymes (BCAT2, BCKDHB, ACADS, HADHA, GSS, GGCT and SLC16A1) and BMI.

\section{Statistical Analysis}


Multivariate data analysis was performed using SIMCA-P+13.0 (Umetrics AB, Umeå, Sweden). GC-MS and LC-MS data sets were scaled to unit variance. Data sets were analyzed using PLS-DA. Metabolite changes responsible for clustering or regression trends within the pattern recognition models were identified by interrogating the corresponding loadings plot. Metabolites identified in the variable importance in projections/coefficients plots were deemed to have changed globally if they contributed to separation in the models with a confidence limit of $95 \% . Q^{2}$ values are shown as a measure of model robustness.

Indirect calorimetry data was analyzed in CaIR and p-values were calculated using ANCOVA / Generalized Linear Model with body mass as a covariate ${ }^{57}$.

All other univariate data, unless otherwise stated, are expressed as means, and error bars depict SEM. A two-tailed Student's t-test, One or Two Way-ANOVA was used to determine $p$ value set as a nominal significance of $p \leq 0.05$.

\section{Declarations}

\section{Acknowledgements}

LDR acknowledges the support of the Diabetes UK RD Lawrence Fellowship (16/0005382), the Biotechnology and Biological Research Council (BB/R013500/1) and the Medical Research Council (MR/R014086/1). JLG is supported by grants from the Medical Research Council (MC_UP_A090_1006; MC_PC_13030; MR/P011705/1 and MR/P01836X/1) and the Biotechnology and Biological Research Council (BB/H013539/2). We thank Yu-Hua Tseng from Harvard University for provision of immortalized human primary adipocytes. RNA-seq analysis was performed by Cambridge Genomic Services.

\section{Author Contributions}

A.W. was directly involved in the majority of experiments. F.N.K. and C.C. performed and analyzed immortalized human adipocyte studies. F.N.K performed mass spectrometry cAMP assays in mouse tissues. J.L.S, A.M and E.B. assisted with experiments throughout. S.A.M, B.D.M, L.D.R and J.L.G assisted with metabolomic screens and ${ }^{13} \mathrm{C}$ isotope studies. G.R.D. and J.D. assisted with quantitative microscopy and open array analysis. A.J.M and A.D.V.M assisted with the design and performance of respirometry. S.V. and A.V-P lead and designed the mouse cold acclimatization studies. J.W. and J.E.S. ran the PET/CT imaging studies. J.G. and K.W. isolated the human adipose biopsies and plasma samples. L.D.R designed and led the studies, interpreted the results and wrote the paper with input from all co-authors.

The authors have no competing interests.

\section{References}


1. Lowell, B.B. \& Spiegelman, B.M. Towards a molecular understanding of adaptive thermogenesis. Nature 404, 652-660 (2000).

2. Wu, Z., et al. Mechanisms controlling mitochondrial biogenesis and respiration through the thermogenic coactivator PGC-1. Cel/ 98, 115-124 (1999).

3. Wu, J., et al. Beige adipocytes are a distinct type of thermogenic fat cell in mouse and human. Cell 150, 366-376 (2012).

4. Wu, J., Cohen, P. \& Spiegelman, B.M. Adaptive thermogenesis in adipocytes: is beige the new brown? Genes Dev 27, 234-250 (2013).

5. Bouillaud, F., Ricquier, D., Thibault, J. \& Weissenbach, J. Molecular approach to thermogenesis in brown adipose tissue: cDNA cloning of the mitochondrial uncoupling protein. Proc Natl Acad Sci US A 82, 445-448 (1985).

6. Oberkofler, H., et al. Uncoupling protein gene: quantification of expression levels in adipose tissues of obese and non-obese humans. J Lipid Res 38, 2125-2133 (1997).

7. Seale, P., et al. Transcriptional control of brown fat determination by PRDM16. Cell Metab 6, 38-54 (2007).

8. Tran, T.T., Yamamoto, Y., Gesta, S. \& Kahn, C.R. Beneficial effects of subcutaneous fat transplantation on metabolism. Cell Metab 7, 410-420 (2008).

9. Liu, X., et al. Brown adipose tissue transplantation improves whole-body energy metabolism. Cell research 23, 851-854 (2013).

10. Kong, X., et al. Brown Adipose Tissue Controls Skeletal Muscle Function via the Secretion of Myostatin. Cell Metab (2018).

11. Feldmann, H.M., Golozoubova, V., Cannon, B. \& Nedergaard, J. UCP1 ablation induces obesity and abolishes diet-induced thermogenesis in mice exempt from thermal stress by living at thermoneutrality. Cell Metab 9, 203-209 (2009).

12. Liu, X., et al. Paradoxical resistance to diet-induced obesity in UCP1-deficient mice. J Clin Invest 111, 399-407 (2003).

13. Enerback, S., et al. Mice lacking mitochondrial uncoupling protein are cold-sensitive but not obese. Nature 387, 90-94 (1997).

14. Lowell, B.B., et al. Development of obesity in transgenic mice after genetic ablation of brown adipose tissue. Nature 366, 740-742 (1993).

15. Hamann, A., Flier, J.S. \& Lowell, B.B. Decreased brown fat markedly enhances susceptibility to dietinduced obesity, diabetes, and hyperlipidemia. Endocrinology 137, 21-29 (1996).

16. Wang, Y.X., et al. Peroxisome-proliferator-activated receptor delta activates fat metabolism to prevent obesity. Cell 113, 159-170 (2003).

17. Palekar, A.G., Tate, S.S., Sullivan, J.F. \& Meister, A. Accumulation of 50oxo-L-proline and 5-oxo-Dproline in the blood plasma in end stage renal disease. Biochemical medicine 14, 339-345 (1975). 
18. Engelke, U.F., et al. NMR spectroscopic studies on the late onset form of 3-methylglutaconic aciduria type I and other defects in leucine metabolism. NMR in biomedicine 19, 271-278 (2006).

19. Avogaro, A. \& Bier, D.M. Contribution of 3-hydroxyisobutyrate to the measurement of 3hydroxybutyrate in human plasma: comparison of enzymatic and gas-liquid chromatography-mass spectrometry assays in normal and in diabetic subjects. J Lipid Res 30, 1811-1817 (1989).

20. Hoffmann, G.F., et al. Physiology and pathophysiology of organic acids in cerebrospinal fluid. Journal of inherited metabolic disease 16, 648-669 (1993).

21. Virtue, S. \& Vidal-Puig, A. Assessment of brown adipose tissue function. Frontiers in physiology 4, 128 (2013).

22. Halestrap, A.P. \& Price, N.T. The proton-linked monocarboxylate transporter (MCT) family: structure, function and regulation. Biochem J 343 Pt 2, 281-299 (1999).

23. Cao, W., et al. p38 mitogen-activated protein kinase is the central regulator of cyclic AMP-dependent transcription of the brown fat uncoupling protein 1 gene. Mol Cell Biol 24, 3057-3067 (2004).

24. Yengo, L., et al. Meta-analysis of genome-wide association studies for height and body mass index in approximately 700000 individuals of European ancestry. Hum Mol Genet 27, 3641-3649 (2018).

25. Rosen, E.D. \& Spiegelman, B.M. What we talk about when we talk about fat. Cell 156, 20-44 (2014).

26. Stanford, K.I., et al. Brown adipose tissue regulates glucose homeostasis and insulin sensitivity. $J$ Clin Invest 123, 215-223 (2013).

27. Villarroya, F., Gavalda-Navarro, A., Peyrou, M., Villarroya, J. \& Giralt, M. The Lives and Times of Brown Adipokines. Trends Endocrinol Metab 28, 855-867 (2017).

28. Roberts, L.D., et al. beta-Aminoisobutyric Acid Induces Browning of White Fat and Hepatic betaOxidation and Is Inversely Correlated with Cardiometabolic Risk Factors. Cell Metab 19, 96-108 (2014).

29. Jang, C., et al. A branched-chain amino acid metabolite drives vascular fatty acid transport and causes insulin resistance. Nat Med 22, 421-426 (2016).

30. Wang, T.J., et al. Metabolite profiles and the risk of developing diabetes. Nat Med 17, 448-453 (2011).

31. Newgard, C.B., et al. A branched-chain amino acid-related metabolic signature that differentiates obese and lean humans and contributes to insulin resistance. Cell Metab 9, 311-326 (2009).

32. Newgard, C.B. Interplay between Lipids and Branched-Chain Amino Acids in Development of Insulin Resistance. Cell Metabolism 15, 606-614 (2012).

33. Roberts, L.D. \& Gerszten, R.E. Toward new biomarkers of cardiometabolic diseases. Cell Metab 18, 43-50 (2013).

34. Roberts, L.D., Koulman, A. \& Griffin, J.L. Towards metabolic biomarkers of insulin resistance and type 2 diabetes: progress from the metabolome. The lancet. Diabetes \& endocrinology 2, 65-75 (2014).

35. Lynch, C.J. \& Adams, S.H. Branched-chain amino acids in metabolic signalling and insulin resistance. Nature reviews. Endocrinology 10, 723-736 (2014). 
36. Herman, M.A., She, P., Peroni, O.D., Lynch, C.J. \& Kahn, B.B. Adipose tissue branched chain amino acid (BCAA) metabolism modulates circulating BCAA levels. J Biol Chem 285, 11348-11356 (2010).

37. Wallace, M., et al. Enzyme promiscuity drives branched-chain fatty acid synthesis in adipose tissues. Nat Chem Biol 14, 1021-1031 (2018).

38. Neinast, M.D., et al. Quantitative Analysis of the Whole-Body Metabolic Fate of Branched-Chain Amino Acids. Cell Metab (2018).

39. Yoneshiro, T., et al. BCAA catabolism in brown fat controls energy homeostasis through SLC25A44. Nature 572, 614-619 (2019).

40. Pietilainen, K.H., et al. Global transcript profiles of fat in monozygotic twins discordant for BMI: pathways behind acquired obesity. PLoS Med 5, e51 (2008).

41. Pfannenberg, C., et al. Impact of age on the relationships of brown adipose tissue with sex and adiposity in humans. Diabetes 59, 1789-1793 (2010).

42. Schulz, N., et al. Role of medium- and short-chain L-3-hydroxyacyl-CoA dehydrogenase in the regulation of body weight and thermogenesis. Endocrinology 152, 4641-4651 (2011).

43. Skilling, $\mathrm{H}$., et al. Brown adipose tissue function in short-chain acyl-CoA dehydrogenase deficient mice. Biochem Biophys Res Commun 400, 318-322 (2010).

44. Carriere, A., et al. Browning of white adipose cells by intermediate metabolites: an adaptive mechanism to alleviate redox pressure. Diabetes 63, 3253-3265 (2014).

45. Lettieri Barbato, D., et al. Glutathione Decrement Drives Thermogenic Program In Adipose Cells. Scientific reports 5, 13091 (2015).

46. Ro, S.H., et al. Sestrin2 inhibits uncoupling protein 1 expression through suppressing reactive oxygen species. Proc Natl Acad Sci U S A 111, 7849-7854 (2014).

47. Liu, D., et al. Activation of mTORC1 is essential for beta-adrenergic stimulation of adipose browning. J Clin Invest 126, 1704-1716 (2016).

48. Liu, D., Ceddia, R.P. \& Collins, S. Cardiac natriuretic peptides promote adipose 'browning' through mTOR complex-1. Molecular metabolism 9, 192-198 (2018).

49. Cao, W., Medvedev, A.V., Daniel, K.W. \& Collins, S. beta-Adrenergic activation of p38 MAP kinase in adipocytes: cAMP induction of the uncoupling protein 1 (UCP1) gene requires p38 MAP kinase. J Biol Chem 276, 27077-27082 (2001).

50. Holecek, M. Beta-hydroxy-beta-methylbutyrate supplementation and skeletal muscle in healthy and muscle-wasting conditions. Journal of cachexia, sarcopenia and muscle 8, 529-541 (2017).

51. Seale, P., et al. Prdm16 determines the thermogenic program of subcutaneous white adipose tissue in mice. J Clin Invest 121, 96-105 (2011).

52. Roberts, L.D., et al. Inorganic nitrate promotes the browning of white adipose tissue through the nitrate-nitrite-nitric oxide pathway. Diabetes 64, 471-484 (2015).

53. Roberts, L.D., et al. The contrasting roles of PPARdelta and PPARgamma in regulating the metabolic switch between oxidation and storage of fats in white adipose tissue. Genome Biol 12, R75 (2011). 
54. Fasshauer, M., et al. Essential role of insulin receptor substrate 1 in differentiation of brown adipocytes. Mol Cell Bio/ 21, 319-329 (2001).

55. Roberts, L.D., et al. Inorganic Nitrate Mimics Exercise-Stimulated Muscular Fiber-Type Switching and Myokine and gamma-Aminobutyric Acid Release. Diabetes 66, 674-688 (2017).

56. Xue, R., et al. Clonal analyses and gene profiling identify genetic biomarkers of the thermogenic potential of human brown and white preadipocytes. Nat Med 21, 760-768 (2015).

57. Mina, A.I., et al. CalR: A Web-Based Analysis Tool for Indirect Calorimetry Experiments. Cell Metab 28, 656-666 e651 (2018).

58. Sasser, T.A., et al. Segmentation and measurement of fat volumes in murine obesity models using Xray computed tomography. Journal of visualized experiments : JoVE, e3680 (2012).

59. Roberts, L.D., Souza, A.L., Gerszten, R.E. \& Clish, C.B. Targeted metabolomics. Curr Protoc Mol Biol Chapter 30, Unit 3032 31-24 (2012).

\section{Figures}


a

Media

transfer

+/- protein denaturation
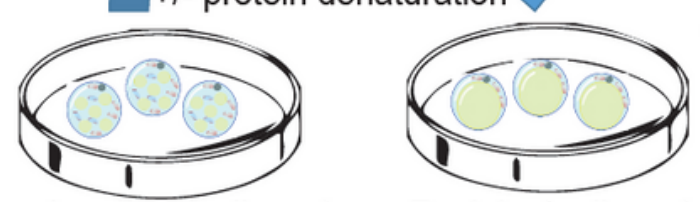

Adipocyte browning

Browning donor adipocytes Recipient adipocytes
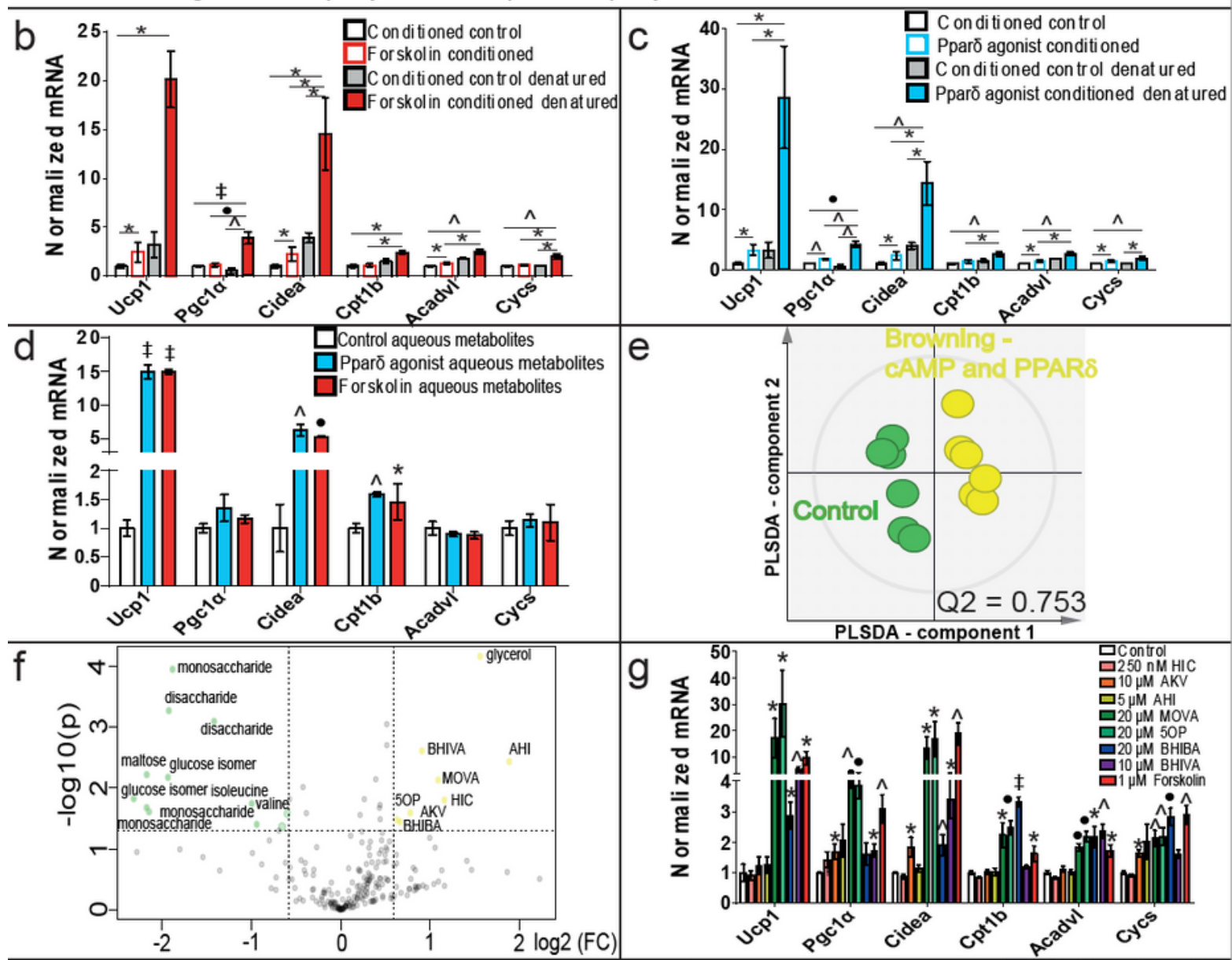

e
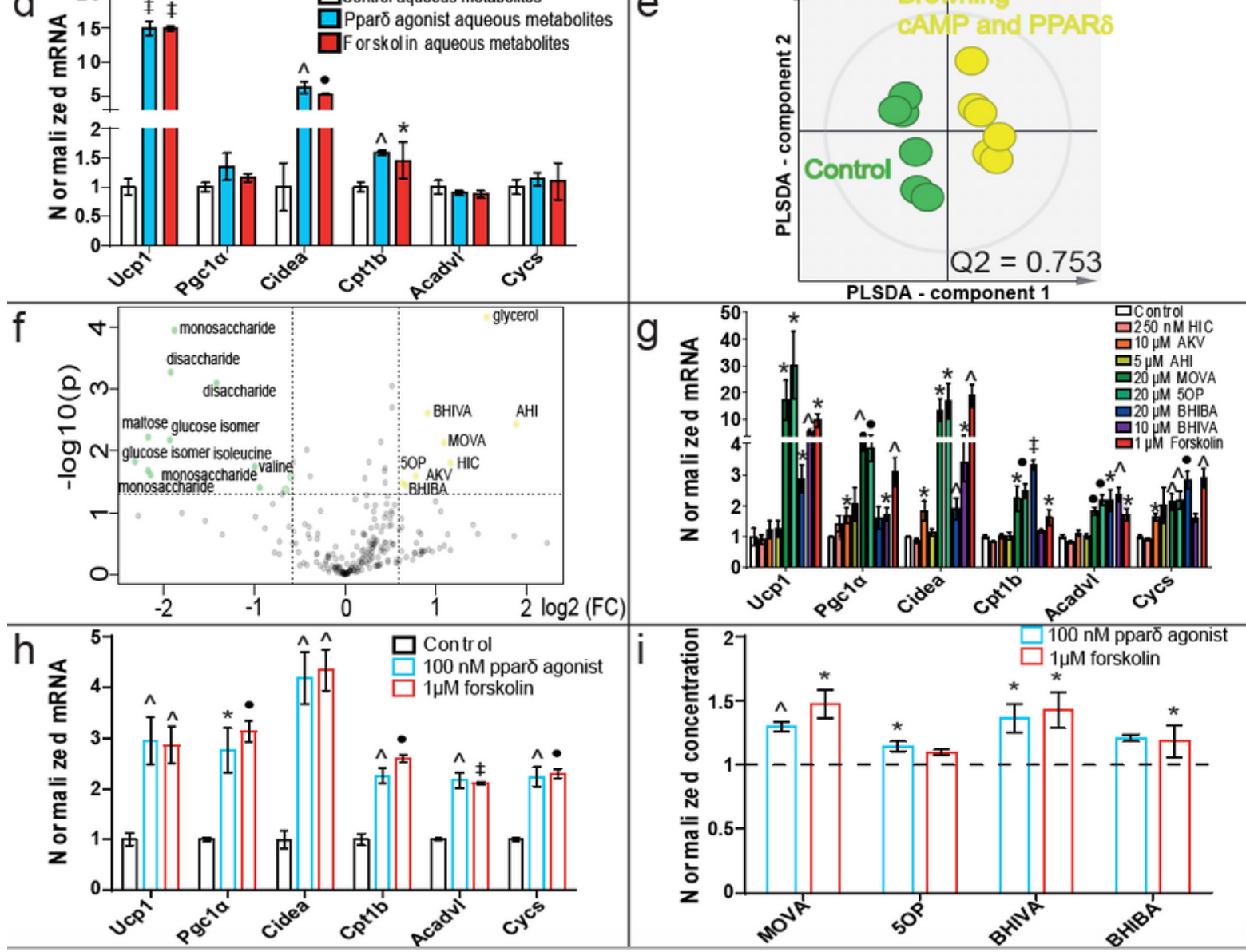

Figure 1

Browning adipocytes secrete metabolites which induce brown adipocyte-associated gene expression in primary adipocytes (a) Brown adipocyte-associated gene expression by murine primary adipocytes after exposure to conditioned media ( \pm protein denaturation) from adipocytes induced to brown through cAMP (forskolin $1 \mu \mathrm{M}$ ) or PPARS (GW0742 $100 \mathrm{nM}$ ). (b, c) Conditioned media from browning adipocytes increases brown adipocyte-associated gene expression in primary adipocytes. Denaturing the media 
protein content enhanced gene expression. (d) Reconstituted aqueous-soluble metabolites from browning adipocyte conditioned media increases brown adipocyte-associated gene expression in primary adipocytes. (e) Metabolomics analysis of the browning adipocyte conditioned media separated from controls in a partial least squares-discriminant analysis (PLSDA) model. (f) Volcano plot analysis of metabolomic data identifies the conditioned media from both browning models was enriched with ahydroxyisocaproic acid (HIC), a-ketoisovaleric acid (AKV), a-hydroxyisovaleric acid (AHI), 3-methyl-2oxovaleric acid (MOVA), 5-oxoproline (5OP), $\beta$-hydroxyisobutyric acid (BHIBA) and $\beta$-hydroxyisovaleric acid (BHIVA). (g) MOVA, 5OP, BHIBA and BHIVA at physiological concentrations increased brown adipocyte-associated gene expression in primary adipocytes. Forskolin treatment given as a positive control for browning. (h) Forskolin or a PPAR $\delta$ agonist induced thermogenic genes in mouse canonical primary brown adipocytes. (i) Conditioned media from primary brown adipocytes treated with either forskolin or PPAR agonist was enriched with MOVA, 5OP, BHIBA and BHIVA. $* p \leq 0.05,{ }^{\wedge} p \leq 0.01, \cdot p \leq$ $0.001, \neq p \leq 0.0001$. Data are mean \pm SEM of at least three biological replicates.
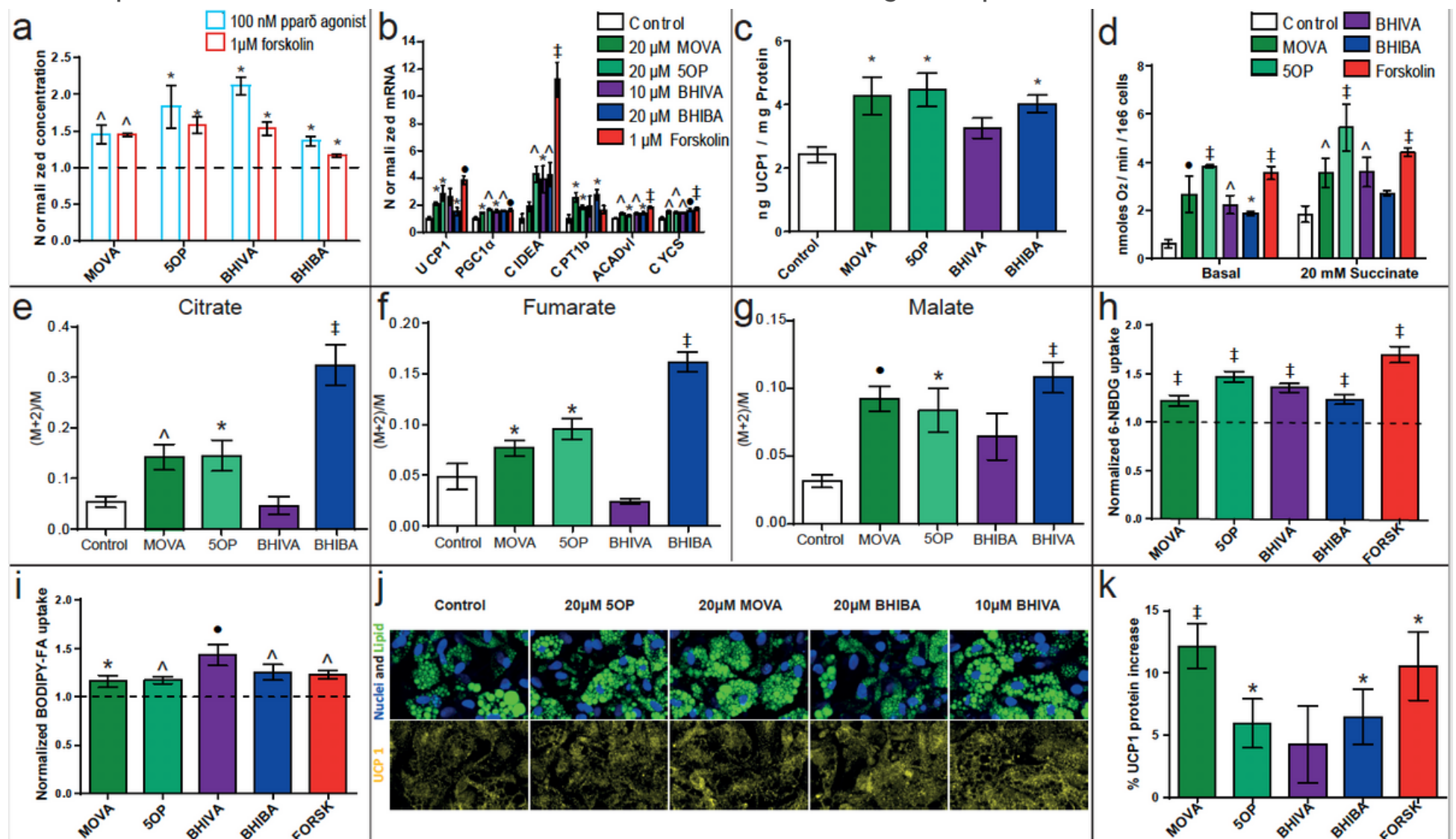

$\mathrm{k}$

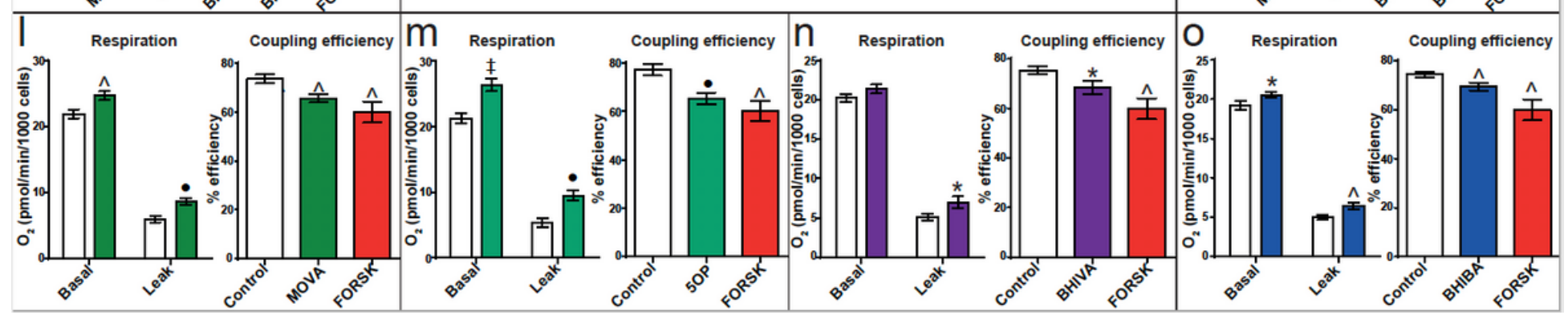

Figure 2 
Browning human adipocytes secrete metabolites which induce a brown adipocyte-like functional phenotype (a) MOVA, 5OP, BHIVA and BHIBA are enriched in browning human adipocyte media. (b) MOVA, 5OP, BHIBA and BHIVA induce brown adipocyte-associated gene expression in human adipocytes. Forskolin treatment given as a positive control for browning. (c) UCP1 protein concentration in human primary adipocytes treated with MOVA, 5OP, BHIVA and BHIBA. (d) Basal and stimulated (succinate 20 $\mathrm{mmol} / \mathrm{L}$ ) oxygen consumption increased in human adipocytes treated with MOVA, 5OP, BHIVA and BHIBA, and Forskolin (provided for comparison). (e - g) TCA cycle intermediates citrate, fumarate and malate 13C-enrichment from 13C-palmitate metabolism in MOVA, 5OP, BHIVA and BHIBA-treated human adipocytes. $M+n$, the isotope of $M$ with an increased $m / z$ of $+n$. (h) Glucose uptake in MOVA, 5OP, BHIVA and BHIBA-treated human adipocytes. Forskolin provided for comparison. (i) Fatty acid uptake in MOVA, 5OP, BHIBA and BHIVA-treated human adipocytes. Forskolin provided for comparison. (j) Composite confocal images (top) of immortalized human adipocytes from neck fat treated with MOVA, 50P, BHIBA and BHIVA, stained for lipid (green), nuclei (blue) and UCP1 (yellow) (bottom). (k) UCP1 in human adipocytes from neck fat treated with MOVA, 5OP, BHIBA, BHIVA or Forskolin (percentage change to control). Basal respiration, proton leak and coupling efficiency, assessed by the Seahorse XF platform Mito Stress assay, in human primary adipocytes isolated from neck fat and treated with MOVA (I) 5OP (m) BHIVA (n) or BHIBA (o) and compared with forskolin. Experiments were performed with $20 \mu \mathrm{M}$ MOVA, $20 \mu \mathrm{M}$ 50P, $20 \mu \mathrm{M}$ BHIBA, $10 \mu \mathrm{M}$ BHIVA and $1 \mu \mathrm{M}$ Forskolin. $* \mathrm{p} \leq 0.05,{ }^{\wedge} \mathrm{p} \leq 0.01, \cdot p \leq 0.001, \neq \mathrm{p} \leq$ 0.0001 . Data are mean \pm SEM of at least three biological replicates. 
a
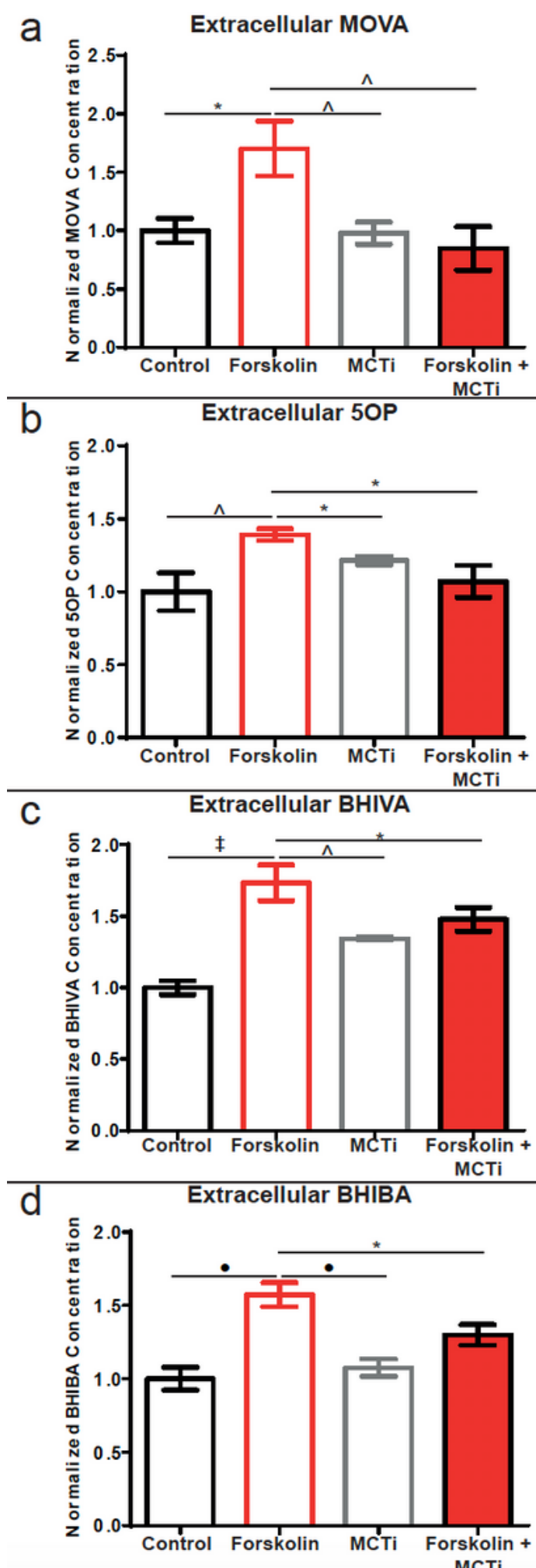
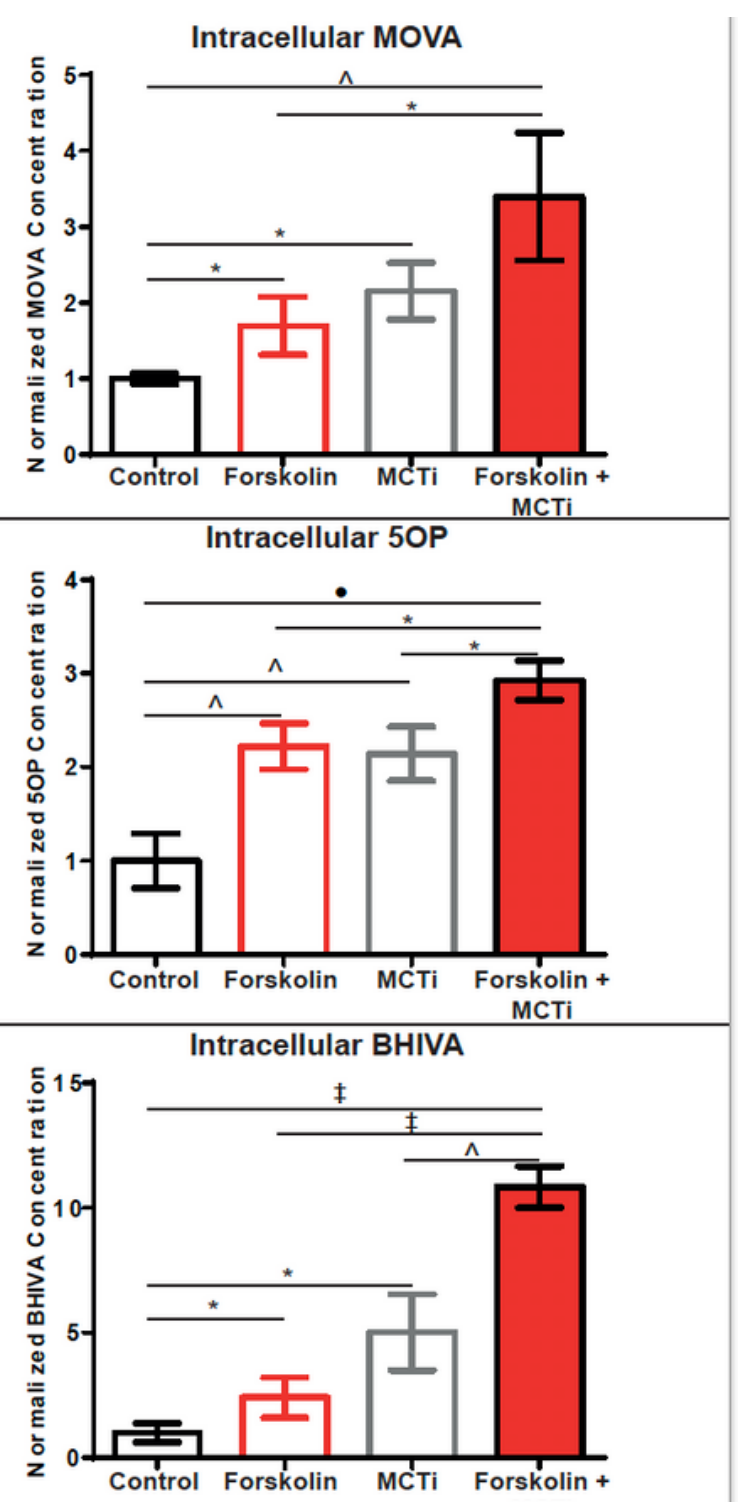

$\mathrm{MCTi}$

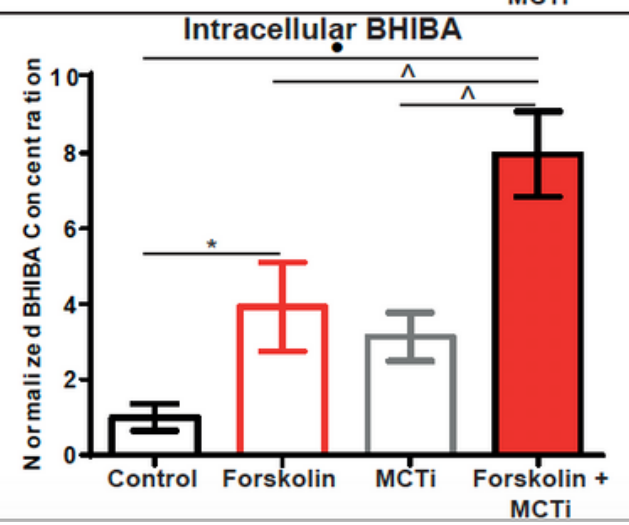

\section{Figure 3}

Export of metabolite signals from browning adipocytes is mediated by monocarboxylate transporters The concentration of (a) MOVA, (b) 5OP, (c) BHIVA and (d) BHIBA measured by liquid chromatography - mass spectrometry in adipocytes (intracellular) and the media (extracellular) of cells treated with either forskolin $(1 \mu \mathrm{M})$, the monocarboxylate transporter inhibitor (MCTi) a-cyano-4-hydroxycinnamate (2 mM) 
or both forskolin and a-cyano-4-hydroxycinnamate. $* p \leq 0.05,{ }^{\wedge} p \leq 0.01, \cdot p \leq 0.001, \neq p \leq 0.0001$. Data are mean \pm SEM of four biological replicates.
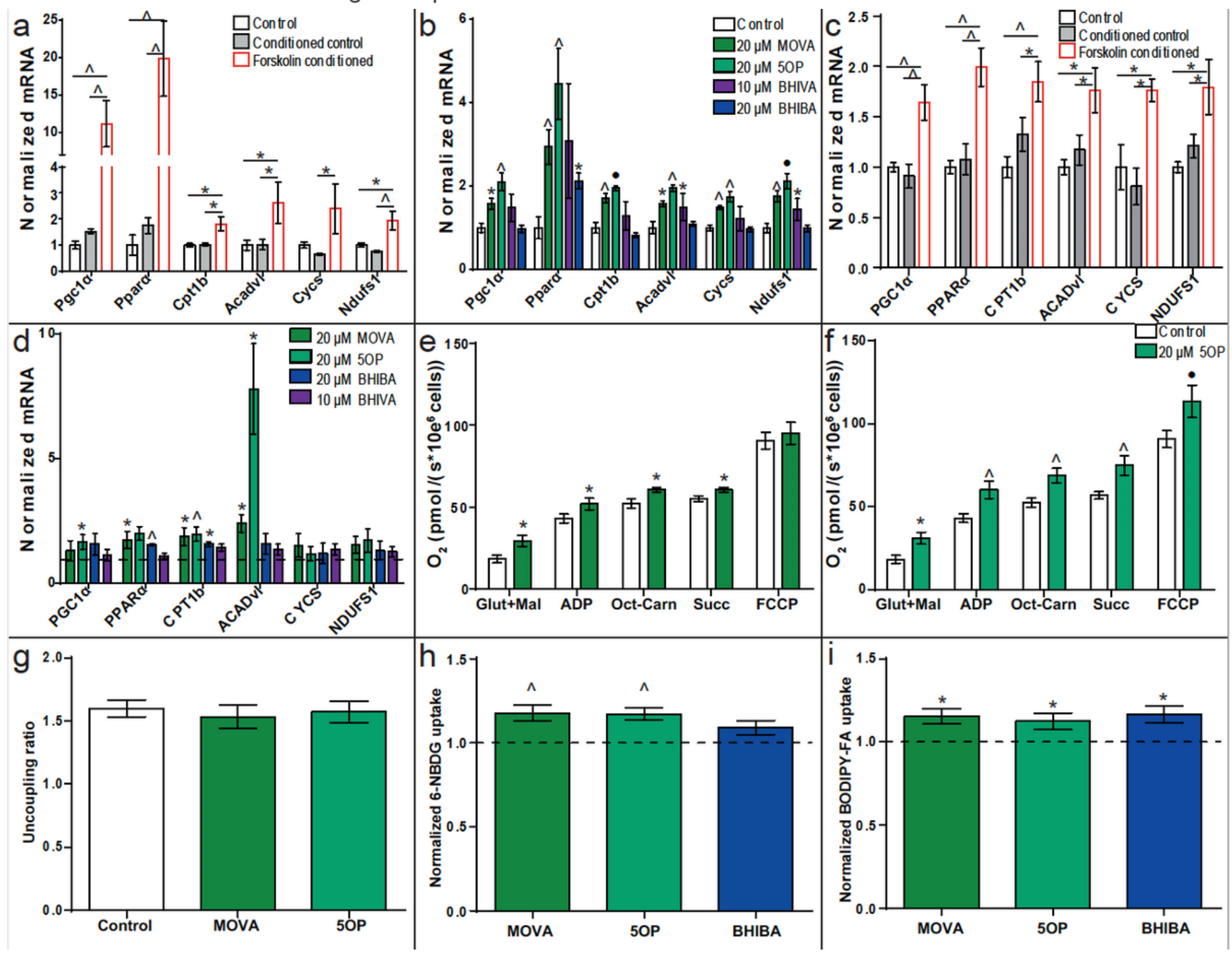

Figure 4

MOVA, 5OP and BHIBA regulate metabolism in mouse and human skeletal myocytes (a) Conditioned media from murine primary adipocytes induced to brown via a cAMP-mediated mechanism increases expression of mitochondrial and metabolic genes in C2C12 myocytes. (b) MOVA and 5OP significantly increase expression of the mitochondrial and metabolic gene panel in $\mathrm{C} 2 \mathrm{C} 12$ myocytes. (c) Conditioned media from browning human primary adipocytes increases expression of mitochondrial and metabolic genes in human primary skeletal myocytes. (d) The expression of the mitochondrial and metabolic gene panel in human primary skeletal myocytes treated with MOVA, 5OP, BHIVA and BHIBA. High-resolution respirometry analysis of basal, complex I (ADP), octanoylcarnitine $\beta$-oxidation (Oct-Carn), Succinatemediated complex II (Succ) and maximal uncoupled electron transport chain (FCCP) respiration in human primary myocytes treated with (e) MOVA or (f) 5OP. (g) The uncoupling ratio of MOVA and 50P-treated human primary skeletal myocytes measured by high-resolution respirometry. (h) Glucose uptake in MOVA 
$(20 \mu \mathrm{M}), 50 \mathrm{P}(20 \mu \mathrm{M})$ and BHIBA $(20 \mu \mathrm{M})$-treated human skeletal myocytes. (i) Fatty acid uptake in MOVA $(20 \mu \mathrm{M}), 50 \mathrm{P}(20 \mu \mathrm{M})$ and BHIBA $(20 \mu \mathrm{M})$-treated human skeletal myocytes. $* \mathrm{p} \leq 0.05,{ }^{\wedge} \mathrm{p} \leq 0.01$, $\cdot p \leq 0.001, \neq p \leq 0.0001$. Data are mean \pm SEM of at least three biological replicates.

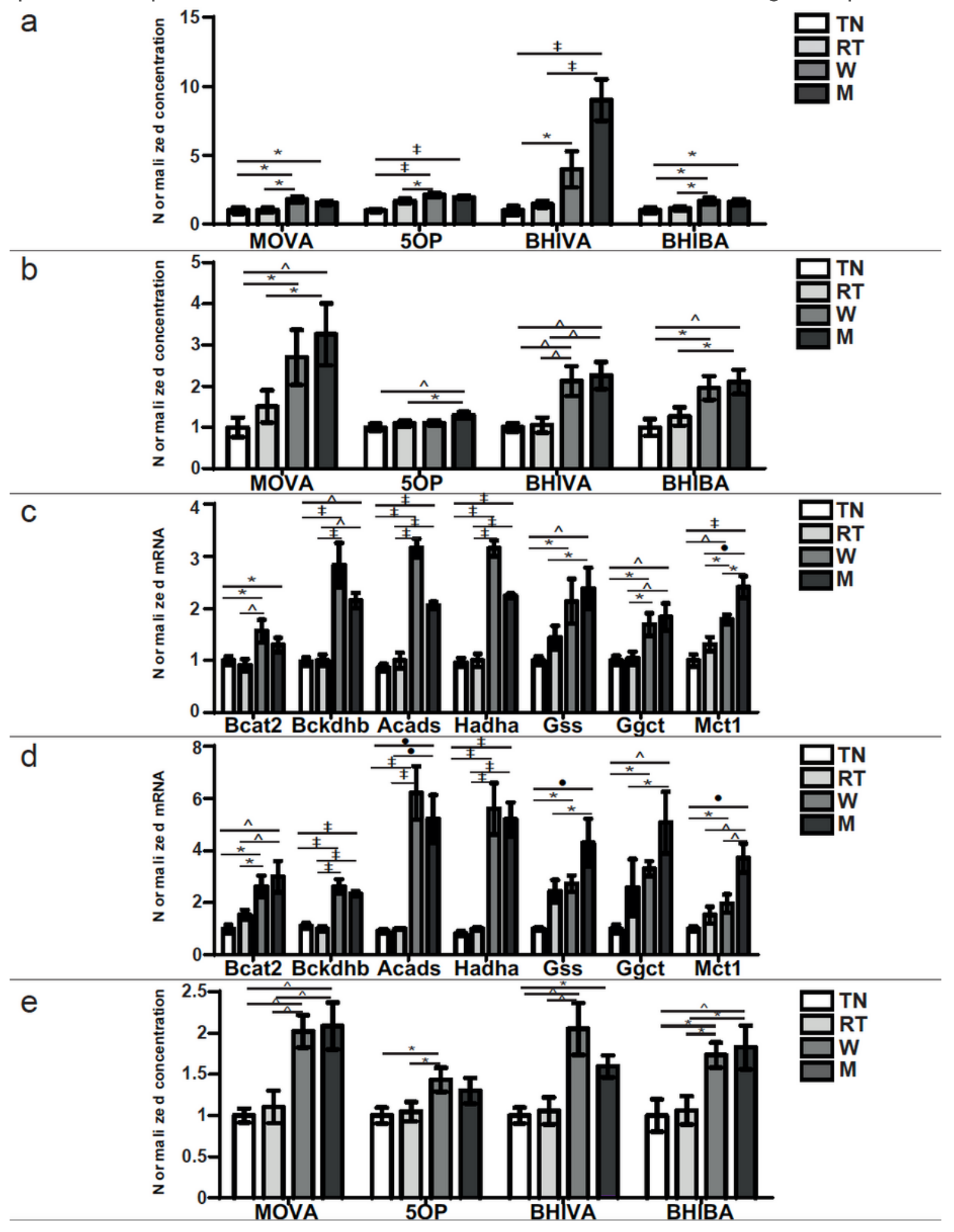

Figure 5

Cold exposure increases adipose tissue and circulating plasma concentrations of the metabokines GCMS analysis of MOVA, 5OP, BHIVA and BHIBA concentration in the (a) interscapular brown adipose tissue 
and (b) subcutaneous inguinal adipose tissue of mice housed at thermoneutrality (TN), room temperature (RT), $8^{\circ} \mathrm{C}$ for 1 week (W) or $8^{\circ} \mathrm{C}$ for 1 month (M). RT-qPCR analysis of branched-chain amino acid catabolic (MOVA, BHIBA and BHIVA biosynthetic) enzymes (Bcat2, Bckdhb, Acads, Hadha), 50P biosynthetic enzymes (Gss, Ggct) and monocarboxylate transporter 1 (Mct1) in the (c) interscapular brown adipose tissue and (d) subcutaneous inguinal adipose tissue of mice housed at thermoneutrality (TN), room temperature (RT), $8^{\circ} \mathrm{C}$ for 1 week (W) or $8^{\circ} \mathrm{C}$ for 1 month (M). (e) MOVA, 5OP, BHIVA and BHIBA concentration in the blood plasma of mice housed at thermoneutrality $(\mathrm{TN})$, room temperature (RT), $8^{\circ} \mathrm{C}$ for 1 week (W) or $8^{\circ} \mathrm{C}$ for 1 month $(M) . * p \leq 0.05,{ }^{\wedge} p \leq 0.01, \cdot p \leq 0.001, \neq p \leq 0.0001$. Data are mean \pm SEM of six biological replicates. 

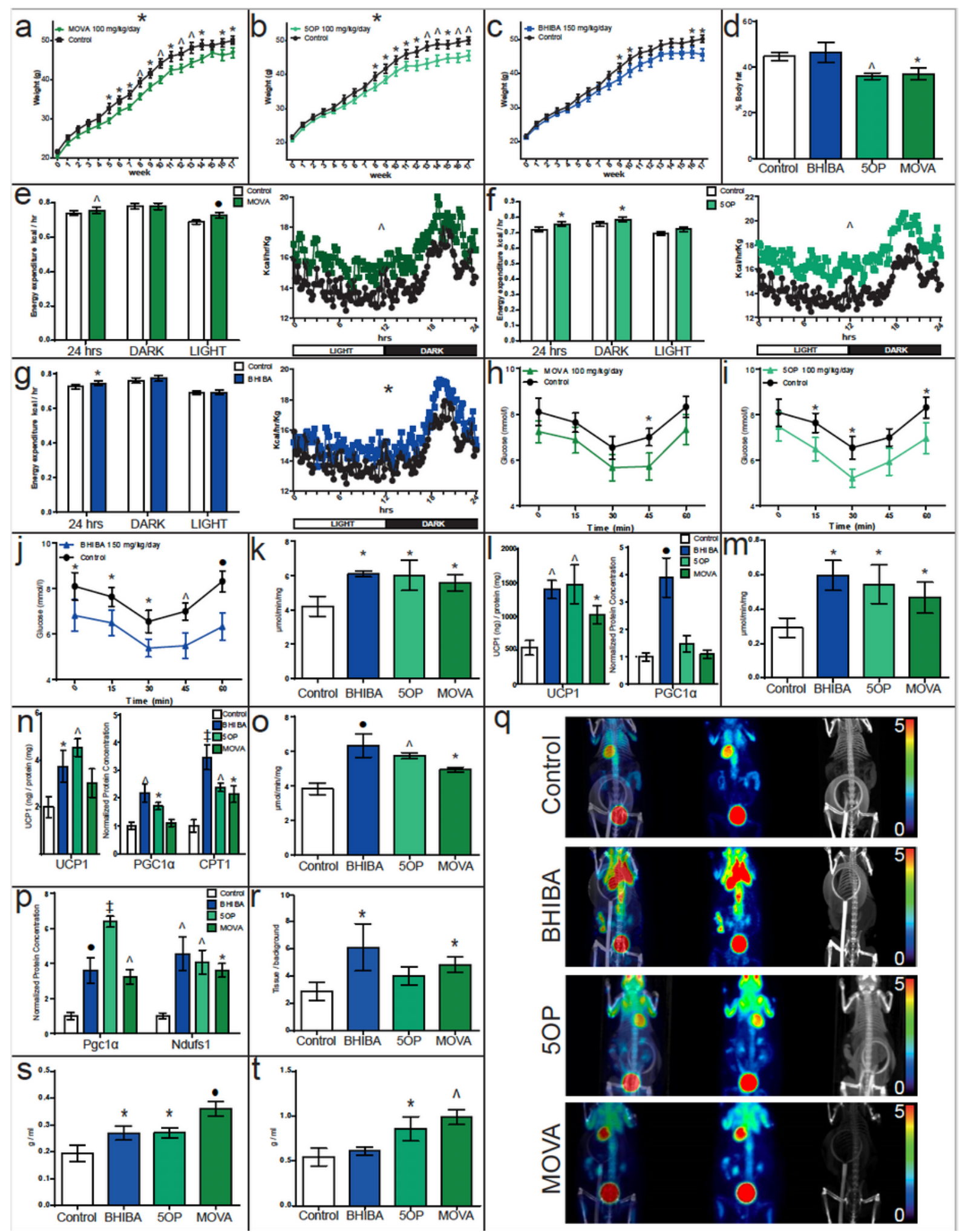

\section{Figure 6}

MOVA, 5OP and BHIBA decrease weight gain and regulate systemic energy metabolism in mice Weights of mice receiving (a) $100 \mathrm{mg} / \mathrm{kg} /$ day MOVA, (b) $100 \mathrm{mg} / \mathrm{kg} /$ day $5 O P$, (c) $150 \mathrm{mg} / \mathrm{kg} /$ day BHIBA compared to controls $(n=10)$. (d) Adiposity of MOVA, 5OP and BHIBA-treated mice. Diurnal weight normalized energy expenditure and average hourly energy expenditure for 24 hour period, 12 hour dark phase (DARK) and 12 hour light phase (LIGHT) of (e) MOVA, (f) 5OP and (g) BHIBA-treated mice $(\mathrm{n}=8)$. 
Insulin tolerance tests of (h) MOVA, (i) 5OP and (j) BHIBA-treated mice $(n=10)$. (k) Increased mitochondrial content in brown adipose tissue (BAT) of BHIBA and 5OP-treated mice $(n=5)$. (l) UCP1 and PGC1a concentration of BAT from BHIBA, 5OP and MOVA-treated mice $(n=5)$. $(m)$ Increased subcutaneous inguinal adipose tissue mitochondrial content in BHIBA and 5OP-treated mice $(n=5)$. $(n)$ UCP1, PGC1a and CPT1 concentration of subcutaneous adipose tissue from BHIBA, 5OP and MOVAtreated mice $(n=5)$. (o) BHIBA, 5OP and MOVA increase soleus muscle mitochondrial content in mice ( $n$ = 5). (p) BHIBA, 5OP and MOVA increase PGC1 $a$ and NDUFS1 in the soleus muscle of mice $(n=5)$. (q) 18F-FDG PET/CT of BHIBA, 5OP and MOVA-treated mice identifies tissue-specific metabolic effects $(\mathrm{n}=$ 5). Scale bars are standardized uptake value (SUV) $\mathrm{g} / \mathrm{ml}$. Right - CT, middle - PET, left - PET/CT. 18FFDG uptake into ( $r$ BAT, and (s) hind limb muscle and ( $\mathrm{t}$ ) forelimb muscle of BHIBA, 5OP and MOVAtreated mice $(n=5) . * p \leq 0.05,{ }^{\wedge} p \leq 0.01, \cdot p \leq 0.001, \neq p \leq 0.0001$. 


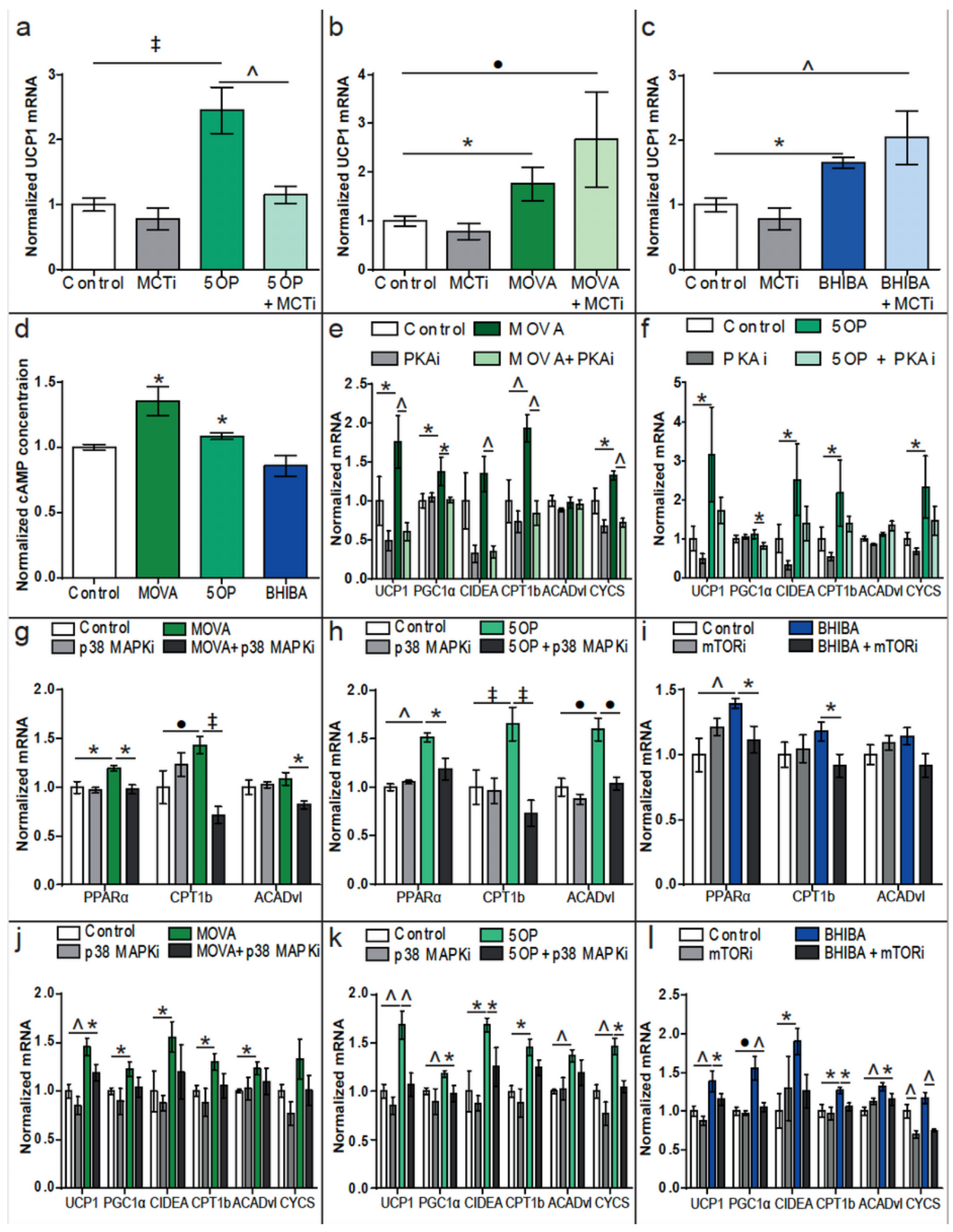

Figure 7

MOVA and 50P signal through cAMP-PKA-p38 MAPK and BHIBA via mTOR The expression of UCP1 in human primary adipocytes treated concomitantly with the monocarboxylate transporter inhibitor a-cyano4-hydroxycinnamate (2 mM), which prevents cellular export and import of monocarboxylate species, and (a) 5OP $(20 \mu \mathrm{M})$, (b) MOVA $(20 \mu \mathrm{M})$ and (c) BHIBA $(20 \mu \mathrm{M})$. (d) Normalized intracellular cAMP concentration measured by Liquid Chromatography-Mass Spectrometry in human primary adipocytes 
treated with MOVA $(20 \mu \mathrm{M}), 50 \mathrm{P}(20 \mu \mathrm{M})$ or BHIBA. Human primary adipocytes treated with the selective Protein Kinase A inhibitor H89 $(10 \mu \mathrm{M})$ (PKAi) with and without (e) $20 \mu \mathrm{M}$ MOVA and (f) $20 \mu \mathrm{M} 50 \mathrm{P}$. Human primary myocytes treated with the p38 MAPK inhibitor Birb796 (500 nM) (p38 MAPKi) with and without (g) $20 \mu \mathrm{M}$ MOVA and (h) $20 \mu \mathrm{M}$ 50P. Inhibition of p38 MAPK signaling abrogates MOVA and 50Pinduced $\beta$-oxidation gene expression in primary human myocytes. (i) Human primary myocytes treated with the mTOR inhibitor temsirolimus (500 nM) (mTORi) with and without $20 \mu \mathrm{M}$ BHIBA. Inhibition of mTOR signaling reduces BHIBA-induced gene expression in primary human myocytes. Human primary adipocytes treated with the p38 MAPK inhibitor Birb796 (500 nM) (p38 MAPKi) with and without (j) 20 $\mu \mathrm{M}$ MOVA and $(\mathrm{k}) 20 \mu \mathrm{M}$ 5OP. Inhibition of p38 MAPK signalling impairs MOVA and 50P-induced brown adipocyte-associated gene expression in primary human adipocytes. (I) Human primary adipocytes treated with the mTOR inhibitor temsirolimus (500 nM) (mTORi) with and without $20 \mu \mathrm{M} \mathrm{BHIBA}$. Inhibition of $\mathrm{mTOR}$ signalling inhibits BHIBA-induced $\beta$-oxidation gene expression in human adipocytes. Data are mean \pm SEM of at least three biological replicates. $* p \leq 0.05, \wedge p \leq 0.01, \cdot p \leq 0.001, \neq p \leq$ 0.0001 .

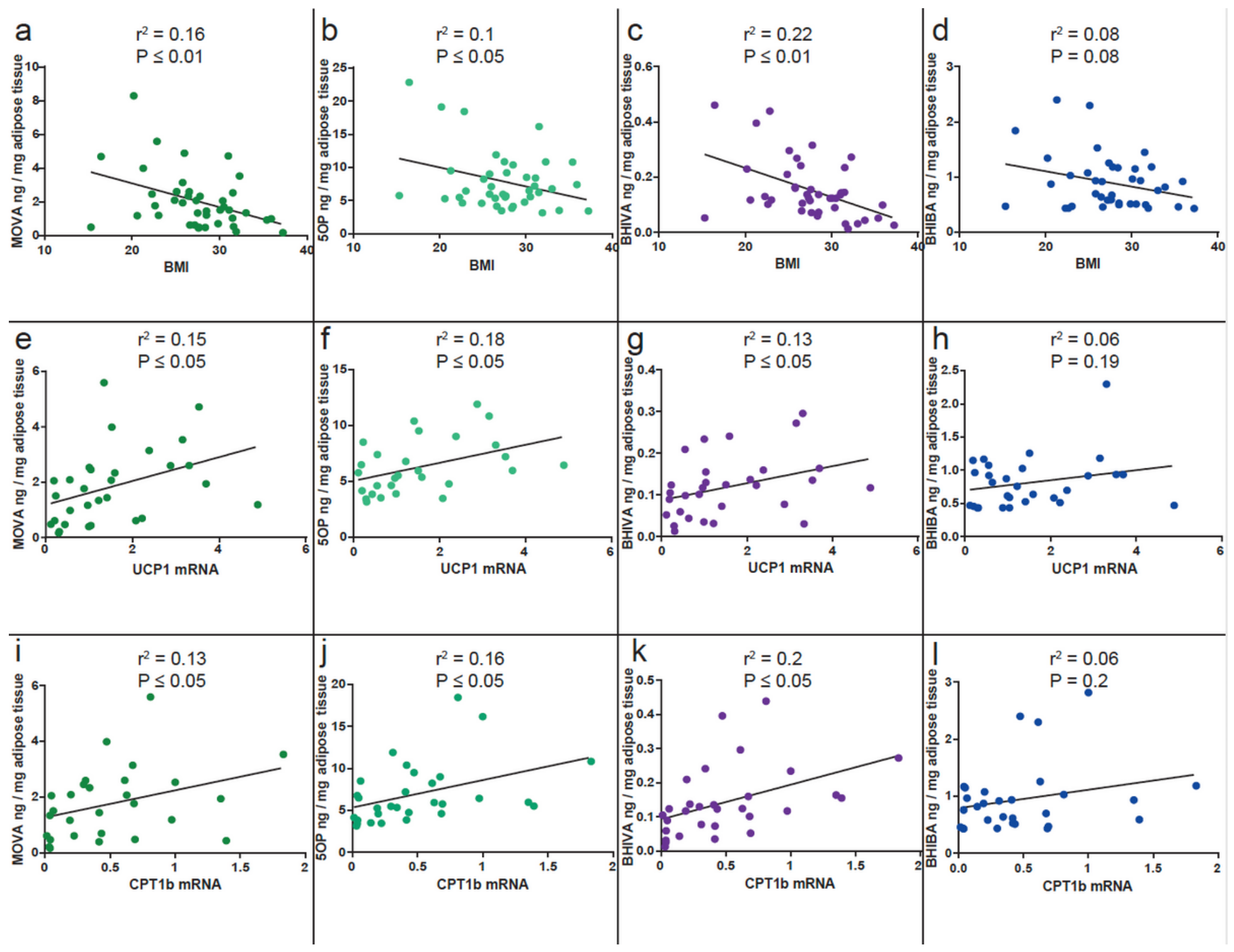

Figure 8 
The adipokine-like metabolite adipose tissue concentrations are inversely correlated with $\mathrm{BMI}$ and positively correlated with brown adipocyte-associated gene expression in humans $(a-d)$ The inverse correlation of MOVA, 5OP, BHIVA and BHIBA subcutaneous adipose tissue concentration, measured by GC-MS, to BMI in human volunteers $(n=42)$. $(e-h)$ Correlation of MOVA, 5OP, BHIVA and BHIBA concentration with UCP1 gene expression in subcutaneous adipose tissue of human volunteers $(n=30)$. $(\mathrm{i}-\mathrm{l})$ Correlation of MOVA, 5OP, BHIVA and BHIBA concentration with CPT1b gene expression in human adipose tissue $(n=30)$.

\section{Supplementary Files}

This is a list of supplementary files associated with this preprint. Click to download.

- SupplementaryInformation2.docx 